\title{
Convergence and quasi-optimality of adaptive FEM for Steklov eigenvalue problems
}

\author{
Eduardo M. Garau Pedro Morin
}

July 17, 2009

\begin{abstract}
In this article we prove convergence of adaptive finite element methods for Steklov eigenvalue problems under very general assumptions for simple as well as multiple eigenvalues starting from any initial triangulation. We also prove the optimality of the approximations assuming Dörfler's Strategy for marking, when we consider simple eigenvalues.
\end{abstract}

Keywords: Steklov Eigenvalue problems; adaptive finite element methods; convergence; optimality.

\section{Introduction}

The main goal of this article is the study of convergence and optimality properties of adaptive finite element methods (AFEM) for Steklov eigenvalue problems. These problems arise, for example, in the analysis of stability of mechanical oscillators immersed in a viscous fluid (see [6] and the references therein), or in the study of vibration modes of a structure interacting with an incompressible fluid [3].

Finite element approximations for these problems have been widely used and analyzed under a general framework. Adaptive finite element methods make an efficient use of the computational resources; for certain problems, it is even indispensable to their numerical resolvability. The ultimate goal of adaptive methods is to equidistribute the error and the computational effort to obtain a sequence of meshes with optimal complexity. Historically, the first step to prove optimality has been to understand convergence of adaptive methods. A basic result of convergence for linear problems has been presented in [17] where very general conditions on the linear problems and on the ingredients of the AFEM that guarantee convergence are stated. Following these ideas, the (plain) convergence of AFEM for elliptic eigenvalue problems was proved in [10]. Optimality of an AFEM using Dörfler's marking strategy [8] has been proved by Stevenson [22] and Cascón et. al. [5] for linear elliptic problems. Linear convergence of an AFEM for elliptic eigenvalue problems has been proved in [14] when considering the approximation of simple eigenvalues, provided the initial mesh is sufficiently fine, and an improvement of this AFEM including optimality is presented in [7]. 
In this article we consider the Steklov eigenvalue problem that consists in finding $\lambda \in \mathbb{R}$, and $u \neq \equiv 0$ such that

$$
-\nabla \cdot(\mathcal{A} \nabla u)+c u=0 \quad \text { in } \Omega, \quad(\mathcal{A} \nabla u) \cdot \vec{n}=\lambda \rho u \quad \text { on } \partial \Omega,
$$

where $\vec{n}$ is the outward unit normal vector of $\Omega$ on $\partial \Omega$, under general assumptions on $\mathcal{A}, c, \rho$ and $\Omega$ that we state precisely in Section 2.1.

This paper contains two main results. The first one, presented in Section 3, is a plain convergence result (without rate) of general adaptive loops (several marking strategies), starting from any initial mesh, valid for single as well as multiple eigenvalues. The second one, presented in Section 5, is a result on the optimal complexity of the sequence of meshes generated by an AFEM using Dörfler's marking Strategy. This second result holds only for simple eigenvalues, whereas the first one holds also for multiple eigenvalues. Both results hold starting from any initial mesh, and with marking strategies that only use the usual a posteriori error estimators, without need of marking due to oscillation terms and without enforcing the so-called interior-node property.

The proof of the convergence result follows [21] and the optimality result is based on the ideas of $[22,5,7]$. Since eigenvalue problems are non-linear it was necessary to adapt those ideas in order to make them applicable to our situation. For instance, a posteriori error estimates usually contain so-called higher-order terms, which, in order to make a rigorous analysis of convergence and optimality, have been taken into account in this article.

\section{Problem statement and approximation}

\section{$2.1 \quad$ Setting}

Let $\Omega \subset \mathbb{R}^{d}$ be a bounded polygonal $(d=2)$ or polyhedral $(d=3)$ domain with a Lipschitz boundary. Let $a, b$ be the bilinear forms defined on $H^{1}(\Omega)$ and $L^{2}(\partial \Omega)$, respectively by

$$
a(u, v):=\int_{\Omega} \mathcal{A} \nabla u \cdot \nabla v+c u v, \quad \text { and } \quad b(u, v):=\int_{\partial \Omega} \rho u v,
$$

where $\mathcal{A}$ is a $W^{1, \infty}(\Omega)$ symmetric-matrix-valued function which is uniformly positive definite, i.e., there exist constants $a_{1}, a_{2}>0$ such that

$$
a_{1}|\xi|^{2} \leq \mathcal{A}(x) \xi \cdot \xi \leq a_{2}|\xi|^{2}, \quad \forall \xi \in \mathbb{R}^{d}, \quad \forall x \in \Omega,
$$

$c \in L^{\infty}(\Omega)$ is a non-negative scalar function such that $\int_{\Omega} c>0$, and $\rho$ is a scalar function such that $0<\rho_{\min } \leq \rho(x) \leq \rho_{\max }$ a.e. in $\partial \Omega$. Without loss of generality, we assume $\int_{\partial \Omega} \rho=1$.

These bilinear forms induce two norms which we denote as

$$
\|v\|_{a}:=a(v, v)^{1 / 2}, \quad v \in H^{1}(\Omega), \quad \text { and } \quad\|v\|_{b}:=b(v, v)^{1 / 2}, \quad v \in L^{2}(\partial \Omega) .
$$

By the assumptions on $\mathcal{A}, c$ and $\rho,\|\cdot\|_{a} \simeq\|\cdot\|_{H^{1}(\Omega)}$ and $\|\cdot\|_{b} \simeq\|\cdot\|_{\partial \Omega}$. Where, hereafter, if $A \subset \partial \Omega$ or $A \subset \Omega,\|\cdot\|_{A}$ denotes the $L^{2}(A)$-norm, i.e.

$$
\|v\|_{A}:=\left(\int_{A}|v|^{2}\right)^{1 / 2} \quad \text { and } \quad\|v\|_{H^{1}(A)}:=\left(\|v\|_{A}^{2}+\|\nabla v\|_{A}^{2}\right)^{1 / 2}
$$


Furthermore, the Trace Theorem implies that there exists a constant $C_{T}>0$ such that

$$
\|u\|_{b} \leq C_{T}\|u\|_{a}, \quad \forall u \in H^{1}(\Omega) .
$$

From now on, we will write $a \lesssim b$ to indicate that $a \leq C b$ with $C>0$ a constant depending on the data of the problem $(\mathcal{A}, c, \rho, \Omega)$, and possibly some shape regularity of the sequence of meshes generated by the adaptive algorithm. Also $a \simeq b$ will indicate that $a \lesssim b$ and $b \lesssim a$.

A weak formulation of the problem stated in the introduction is thus:

Steklov Eigenproblem. Find $\lambda \in \mathbb{R}$ and $u \in H^{1}(\Omega)$ satisfying

$$
\left\{\begin{array}{l}
a(u, v)=\lambda b(u, v), \quad \forall v \in H^{1}(\Omega), \\
\|u\|_{b}=1
\end{array}\right.
$$

Under our assumptions on $\mathcal{A}, c$ and $\rho$, following the lines along [2] for a slightly different situation, it can be proved that problem (2) has a countable sequence of eigenvalues

$$
0<\lambda_{1} \leq \lambda_{2} \leq \lambda_{3} \leq \ldots \nearrow \infty
$$

and corresponding eigenfunctions $u_{1}, u_{2}, u_{3}, \ldots$ which can be assumed to satisfy

$$
b\left(u_{i}, u_{j}\right)=\delta_{i j}:=\left\{\begin{array}{ll}
1 & \text { if } i=j, \\
0 & \text { if } i \neq j,
\end{array} \quad \text { and } \quad a\left(u_{i}, u_{j}\right)= \begin{cases}\lambda_{i} & i=j \\
0 & i \neq j\end{cases}\right.
$$

where in the sequence $\left\{\lambda_{j}\right\}_{j \in \mathbb{N}}$, the $\lambda_{j}$ 's are repeated according to geometric multiplicity.

Also, the eigenvalues can be characterized as extrema of the Rayleigh quotient

$$
\Lambda(u):=\frac{a(u, u)}{b(u, u)}
$$

by the following Minimum-Maximum Principle:

$$
\lambda_{i}=\min _{\substack{V_{i} \subset H^{1}(\Omega) \backslash H_{0}^{1}(\Omega) \\ \operatorname{dim} V_{i}=i}} \max _{u \in V_{i}} \Lambda(u)=\max _{u \in \operatorname{span}\left\{u_{1}, \ldots, u_{i}\right\}} \Lambda(u), \quad i=1,2, \ldots
$$

It will be important in the analysis of convergence to use the following regularity result.

Remark 2.1 (Regularity of eigenfunctions). For $w \in L^{2}(\partial \Omega)$, let $u \in H^{1}(\Omega)$ be the solution of the elliptic Neumann problem

$$
a(u, v)=b(w, v), \quad \forall v \in H^{1}(\Omega) .
$$

If $\bar{c}>0$ denotes the mean value of $c$ in $\Omega$, then in the weak sense

$$
-\nabla \cdot(\mathcal{A} \nabla u)+\bar{c} u=f, \quad \text { in } \Omega \quad(\mathcal{A} \nabla u) \cdot \vec{n}=g, \quad \text { on } \partial \Omega,
$$

where $f:=-(c-\bar{c}) u$ and $g:=\rho w$. As a consequence of Theorem 4 in [18] and the Closed Graph Theorem, we have that $u \in H^{1+r}(\Omega)$ for all $r \in(0,1 / 2)$ and

$$
\|u\|_{H^{1+r}(\Omega)} \lesssim\|f\|_{L^{2}(\Omega)}+\|g\|_{L^{2}(\partial \Omega)}
$$


Since $\|f\|_{L^{2}(\Omega)}=\|(c-\bar{c}) u\|_{L^{2}(\Omega)} \lesssim\|u\|_{L^{2}(\Omega)} \lesssim\|u\|_{a} \lesssim\|w\|_{b}$ and $\|g\|_{L^{2}(\partial \Omega)} \lesssim$ $\|w\|_{b}$ we obtain for the solution $u$ of the elliptic Neumann problem (3) that

$$
\|u\|_{H^{1+r}(\Omega)} \lesssim\|w\|_{b}, \quad \text { for all } r \in(0,1 / 2) .
$$

Finally, since the eigenfunctions of the Steklov eigenvalue problem (2) are solutions of the elliptic Neumann problem (3) with $w:=\lambda u$, we have that they belong to $H^{1+r}(\Omega)$, for all $r \in(0,1 / 2)$. Here the constants involved in the inequalities also depend on $r$, which from now on will be kept fixed at any value in $(0,1 / 2)$.

\subsection{Discrete problem}

In order to define the discrete approximations to the continuous problem (2) we will consider triangulations of the domain $\Omega$. Let $\mathcal{T}_{0}$ be an initial conforming triangulation of $\Omega$, that is, a partition of $\Omega$ into $d$-simplices such that if two elements intersect, they do so at a full vertex/edge/face of both elements. Let $\mathbb{T}$ denote the set of all conforming triangulations of $\Omega$ obtained from $\mathcal{T}_{0}$ by refinement using the newest vertex bisection procedure in two dimensions and the bisection procedure of Kossaczký in three dimensions [19] which coincide (after some re-labeling) with those presented by Stevenson [23].

For any triangulation $\mathcal{T} \in \mathbb{T}, \mathcal{S}$ will denote the set of sides, where by side we mean an edge if $d=2$ and a face if $d=3$. Moreover, $\mathcal{S}_{\Omega}$ will denote the set of interior sides and $\mathcal{S}_{\partial \Omega}$ will denote the set of boundary sides.

Due to the processes of refinement used, the family $\mathbb{T}$ is shape regular, i.e.,

$$
\sup _{\mathcal{T} \in \mathbb{T}} \sup _{T \in \mathcal{T}} \frac{\operatorname{diam}(T)}{\rho_{T}}=: \kappa_{\mathbb{T}}<\infty,
$$

where $\operatorname{diam}(T)$ is the diameter of $T$, and $\rho_{T}$ is the radius of the largest ball contained in it. Throughout this article, we only consider meshes $\mathcal{T}$ that belong to the family $\mathbb{T}$, so the shape regularity of all of them is bounded by the uniform constant $\kappa_{\mathbb{T}}$ which only depends on the initial triangulation $\mathcal{T}_{0}$ [19]. Also, the diameter of any element $T \in \mathcal{T}$ is equivalent to the local meshsize $h_{T}:=|T|^{1 / d}$, which in turn defines the global meshsize $h_{\mathcal{T}}:=\max _{T \in \mathcal{T}} h_{T}$.

Let $\ell \in \mathbb{N}$ be fixed, and let $\mathbb{V}_{\mathcal{T}}$ be the finite element space consisting of continuous functions which are polynomials of degree $\leq \ell$ in each element of $\mathcal{T}$, i.e,

$$
\mathbb{V}_{\mathcal{T}}:=\left\{v \in H^{1}(\Omega):\left.\quad v\right|_{T} \in \mathcal{P}_{\ell}(T), \quad \forall T \in \mathcal{T}\right\} .
$$

By definition, $\mathbb{V}_{\mathcal{T}} \subset H^{1}(\Omega)$ and if $\mathcal{T}_{*}$ is a refinement of $\mathcal{T}$, then $\mathbb{V}_{\mathcal{T}} \subset \mathbb{V}_{\mathcal{T}_{*}}$.

We consider the following approximation of the continuous eigenvalue problem (2):

Discrete Steklov Eigenproblem. Find $\lambda_{\mathcal{T}} \in \mathbb{R}$ and $u_{\mathcal{T}} \in \mathbb{V}_{\mathcal{T}}$ such that

$$
\left\{\begin{array}{l}
a\left(u_{\mathcal{T}}, v_{\mathcal{T}}\right)=\lambda_{\mathcal{T}} b\left(u_{\mathcal{T}}, v_{\mathcal{T}}\right), \quad \forall v_{\mathcal{T}} \in \mathbb{V}_{\mathcal{T}}, \\
\left\|u_{\mathcal{T}}\right\|_{b}=1 .
\end{array}\right.
$$

Analogously to the continuous problem (2), it can be proved that the discrete problem (5) has a finite sequence of eigenvalues

$$
0<\lambda_{1, \mathcal{T}} \leq \lambda_{2, \mathcal{T}} \leq \lambda_{3, \mathcal{T}} \leq \ldots \leq \lambda_{N_{\mathcal{T}}^{\partial}, \mathcal{T}},
$$


where $N_{\mathcal{T}}^{\partial}$ is the number of nodes which lie on $\partial \Omega$, and corresponding eigenfunctions $u_{1, \mathcal{T}}, u_{2, \mathcal{T}}, u_{3, \mathcal{T}}, \ldots, u_{N_{T}^{\partial}, \mathcal{T}}$, which can be assumed to satisfy

$$
b\left(u_{i, \mathcal{T}}, u_{j, \mathcal{T}}\right)=\delta_{i j} .
$$

Moreover, we have the following Minimum-Maximum Principle:

$$
\lambda_{i, \mathcal{T}}=\min _{\substack{V_{i, \mathcal{T}} \subset \mathbb{V}_{\mathcal{T}} \backslash H_{0}^{1}(\Omega) \\ \operatorname{dim} V_{i, \mathcal{T}}=i}} \max _{u \in V_{i, \mathcal{T}}} \Lambda(u)=\max _{u \in \operatorname{span}\left\{u_{1, \mathcal{T}}, \ldots, u_{i, \mathcal{T}}\right\}} \Lambda(u), \quad i=1,2, \ldots, N_{\mathcal{T}}^{\partial} .
$$

As a direct consequence of this principle, if $\mathcal{T}_{*}$ is any refinement of $\mathcal{T}$,

$$
\lambda_{i} \leq \lambda_{i, \mathcal{T}_{*}} \leq \lambda_{i, \mathcal{T}}, \quad i=1,2, \ldots, N_{\mathcal{T}}^{\partial} .
$$

\subsection{Approximation notion}

Since an eigenvalue has at least two eigenfunctions, we need to define a notion of approximation error. This notion allows us to perform an error analysis taking into account the fact that existing codes for computing (discrete) eigenvalues just return one eigenfunction corresponding to the desired eigenvalue, but not necessarily the one closest to the continuous eigenfunction that we may want to approximate. Moreover, it is usually the case that the discrete eigenvalues corresponding to a repeated continuous eigenvalue are not equal.

From now on, we consider the approximation of a certain fixed eigenvalue $\lambda=\lambda_{j_{0}}$ of the continuous problem (2), $j_{0} \in \mathbb{N}$, and let $j \in \mathbb{N}$ denote the index that satisfies $\lambda=\lambda_{j}=\lambda_{j+1}=\ldots=\lambda_{j+R-1}<\lambda_{j+R}$, and that $\lambda_{j-1}<\lambda_{j}$, if $j>1$, i.e., $\lambda$ is an eigenvalue with multiplicity $R \geq 1$, and $j$ is the smallest index that satisfies $\lambda_{j_{0}}=\lambda=\lambda_{j}$. It is worth noticing that we do not assume the knowledge of $j$ or $R$ for the statement of the adaptive algorithm, but we define them in order to state and prove our results precisely.

Definition 2.2 (Eigenspaces). We denote by $E_{\lambda}$ the $R$-dimensional space of eigenfunctions of the continuous problem (2) associated to $\lambda$, i.e.,

$$
E_{\lambda}:=\left\{u \in H^{1}(\Omega) \mid a(u, v)=\lambda b(u, v), \quad \forall v \in H^{1}(\Omega)\right\},
$$

and the $R$-dimensional approximation space $E_{\lambda}^{\mathcal{T}} \subset \mathbb{V}_{\mathcal{T}}$ is defined by

$$
E_{\lambda}^{\mathcal{T}}:=\sum_{i=j}^{j+R-1} E_{\lambda_{i, \mathcal{T}}}^{\mathcal{T}},
$$

where $E_{\lambda_{i, \mathcal{T}}^{\mathcal{T}}}^{\mathcal{T}}:=\left\{u_{\mathcal{T}} \in \mathbb{V}_{\mathcal{T}} \mid a\left(u_{\mathcal{T}}, v_{\mathcal{T}}\right)=\lambda_{i, \mathcal{T}} b\left(u_{\mathcal{T}}, v_{\mathcal{T}}\right), \forall v_{\mathcal{T}} \in \mathbb{V}_{\mathcal{T}}\right\}$ is the space of eigenfunctions of the discrete problem (5) associated to $\lambda_{i, \mathcal{T}}$. We also define the normalized sets

$$
\tilde{E}_{\lambda}:=\left\{u \in E_{\lambda} \mid\|u\|_{b}=1\right\}, \quad \text { and } \quad \tilde{E}_{\lambda}^{\mathcal{T}}:=\left\{u_{\mathcal{T}} \in E_{\lambda}^{\mathcal{T}} \mid\left\|u_{\mathcal{T}}\right\|_{b}=1\right\} .
$$

Remark 2.3. If $v \in H^{1}(\Omega)$ and $u \in \tilde{E}_{\lambda}$ then the following statements are equivalent:

(i) $u=\arg \max _{\tilde{u} \in \tilde{E}_{\lambda}} a(\tilde{u}, v)$. 
(ii) $u=\arg \max _{\tilde{u} \in \tilde{E}_{\lambda}} b(\tilde{u}, v)$.

(iii) The $a$-distance from $v$ to $\tilde{E}_{\lambda}$ is achieved by $u$; i.e., $\|u-v\|_{a}=\operatorname{dist}_{a}\left(v, \tilde{E}_{\lambda}\right)$.

(iv) The $b$-distance from $v$ to $\tilde{E}_{\lambda}$ is achieved by $u$; i.e., $\|u-v\|_{b}=\operatorname{dist}_{b}\left(v, \tilde{E}_{\lambda}\right)$.

In order to prove this remark, it is sufficient to observe that if $\tilde{u} \in \tilde{E}_{\lambda}$, then

$$
\begin{gathered}
a(\tilde{u}, v)=\lambda b(\tilde{u}, v), \quad \text { for all } v \in H^{1}(\Omega), \\
a(\tilde{u}, v) \leq a(u, v) \Longleftrightarrow-2 a(u, v) \leq-2 a(\tilde{u}, v) \Longleftrightarrow\|u-v\|_{a} \leq\|\tilde{u}-v\|_{a},
\end{gathered}
$$

and

$$
b(\tilde{u}, v) \leq b(u, v) \Longleftrightarrow-2 b(u, v) \leq-2 b(\tilde{u}, v) \Longleftrightarrow\|u-v\|_{b} \leq\|\tilde{u}-v\|_{b} .
$$

Definition 2.4. We define $\tilde{\mathcal{P}}_{\lambda}: H^{1}(\Omega) \rightarrow \tilde{E}_{\lambda}$ as the non-linear projection operator onto $\tilde{E}_{\lambda}$, more precisely, for $v \in H^{1}(\Omega)$, the projection $\tilde{\mathcal{P}}_{\lambda} v$ is the element in $\tilde{E}_{\lambda}$ satisfying

$$
\operatorname{dist}_{a}\left(v, \tilde{E}_{\lambda}\right)=\left\|v-\tilde{\mathcal{P}}_{\lambda} v\right\|_{a},
$$

(or $\operatorname{dist}_{b}\left(v, \tilde{E}_{\lambda}\right)=\left\|v-\tilde{\mathcal{P}}_{\lambda} v\right\|_{b}$, due to Remark 2.3). Given $v \in H^{1}(\Omega)$, typically $v \in \mathbb{V}_{\mathcal{T}}$ for some triangulation $\mathcal{T}$, $\operatorname{dist}_{a}\left(v, \tilde{E}_{\lambda}\right)$ will be our notion of error for the eigenfunction.

We finish this section by noticing that the a priori error estimates stated in [24] and [13] guarantee that if the mesh $\mathcal{T}$ is sufficiently fine, there holds that

$$
0 \leq \lambda_{j+i, \mathcal{T}}-\lambda \lesssim h_{\mathcal{T}}^{2 r}, \quad \text { for } i=0,1,2, \ldots, R-1 .
$$

\subsection{A posteriori error estimators}

A posteriori estimates for Steklov eigenvalue problems have been recently studied by Armentano and Padra [1] for $d=2$ and Zuppa [25] for $d=3$. In this section we present the residual type a posteriori estimates for eigenvalue problems and state some already known properties.

Given an interior side $S \in \mathcal{S}_{\Omega}$, we denote by $T_{1}$ and $T_{2}$ the elements in $\mathcal{T}$ which share $S$, and by $\overrightarrow{n_{i}}$ the outward unit normal vector of $T_{i}$ on $S$, for $i=1,2$. On the other hand, if $S \in \mathcal{S}_{\partial \Omega}$ is a boundary side, we denote by $\vec{n}$ the outward unit normal vector on $S$.

For $S \in \mathcal{S}$ we define $\omega_{\mathcal{T}}(S)$ as the union of the elements in $\mathcal{T}$ sharing $S$. For $T \in \mathcal{T}, \mathcal{N}_{\mathcal{T}}(T):=\left\{T^{\prime} \in \mathcal{T}: T^{\prime} \cap T \neq \emptyset\right\}$ denotes the set of neighbors of $T$ in $\mathcal{T}$, and $\omega_{\mathcal{T}}(T):=\bigcup_{T^{\prime} \in \mathcal{N}_{\mathcal{T}}(T)} T^{\prime}$, the patch of neighbors of $T$ in $\mathcal{T}$.

Definition 2.5 (Residual, element residual and jump residual). For a generic function $v \in H^{1}(\Omega) \backslash H_{0}^{1}(\Omega)$, we define the Residual $\mathbf{R}(v) \in\left(H^{1}(\Omega)\right)^{\prime}$ (the topological dual space of $\left.H^{1}(\Omega)\right)$ by

$$
\langle\mathbf{R}(v), w\rangle:=a(v, w)-\Lambda(v) b(v, w), \quad \text { for all } w \in H^{1}(\Omega) .
$$

For discrete functions $v_{\mathcal{T}} \in \mathbb{V}_{\mathcal{T}}$ we define the element residual $R\left(v_{\mathcal{T}}\right)$ by

$$
\left.R\left(v_{\mathcal{T}}\right)\right|_{T}:=-\nabla \cdot\left(\mathcal{A} \nabla v_{\mathcal{T}}\right)+c v_{\mathcal{T}},, \quad \text { for all } T \in \mathcal{T},
$$


and the jump residual $J\left(v_{\mathcal{T}}\right)$ by

$$
\left.J\left(v_{\mathcal{T}}\right)\right|_{S}:= \begin{cases}\frac{1}{2}\left[\left.\left(\mathcal{A} \nabla v_{\mathcal{T}}\right)\right|_{T_{1}} \cdot \overrightarrow{n_{1}}+\left.\left(\mathcal{A} \nabla v_{\mathcal{T}}\right)\right|_{T_{2}} \cdot \overrightarrow{n_{2}}\right], & \text { if } S \in \mathcal{S}_{\Omega}, \\ \left(\mathcal{A} \nabla v_{\mathcal{T}}\right) \cdot \vec{n}-\Lambda\left(v_{\mathcal{T}}\right) \rho v_{\mathcal{T}}, & \text { if } S \in \mathcal{S}_{\partial \Omega}\end{cases}
$$

Remark 2.6. For $v_{\mathcal{T}} \in \mathbb{V}_{\mathcal{T}}$, the Residual $\mathbf{R}\left(v_{\mathcal{T}}\right)$ is related to the element residual $R\left(v_{\mathcal{T}}\right)$ and the jump residual $J\left(v_{\mathcal{T}}\right)$ through the following identity:

$$
\left\langle\mathbf{R}\left(v_{\mathcal{T}}\right), w\right\rangle=\sum_{T \in \mathcal{T}}\left(\int_{T} R\left(v_{\mathcal{T}}\right) w+\int_{\partial T} J\left(v_{\mathcal{T}}\right) w\right), \quad \forall w \in H^{1}(\Omega),
$$

which is obtained integrating by parts elementwise.

Definition 2.7 (Local and global error estimator). For $v_{\mathcal{T}} \in \mathbb{V}_{\mathcal{T}}$ we define the local error estimator $\eta_{\mathcal{T}}\left(v_{\mathcal{T}} ; T\right)$ by

$$
\eta_{\mathcal{T}}^{2}\left(v_{\mathcal{T}} ; T\right):=h_{T}^{2}\left\|R\left(v_{\mathcal{T}}\right)\right\|_{T}^{2}+h_{T}\left\|J\left(v_{\mathcal{T}}\right)\right\|_{\partial T}^{2}, \quad \text { for all } T \in \mathcal{T},
$$

and the global error estimator $\eta_{\mathcal{T}}\left(v_{\mathcal{T}}\right)$ is given by $\eta_{\mathcal{T}}^{2}\left(v_{\mathcal{T}}\right):=\sum_{T \in \mathcal{T}} \eta_{\mathcal{T}}^{2}\left(v_{\mathcal{T}} ; T\right)$.

Whenever $\Xi$ is a subset of $\mathcal{T}, \eta_{\mathcal{T}}^{2}\left(v_{\mathcal{T}} ; \Xi\right)$ will denote the sum $\sum_{T \in \Xi} \eta_{\mathcal{T}}^{2}\left(v_{\mathcal{T}} ; T\right)$.

The following upper bound for the residual can be easily proved by using the Clément or the Scott-Zhang interpolation operator [20], details can be found in [1].

Theorem 2.8 (Upper Bound for the Residual). If $\left(\lambda_{\mathcal{T}}, u_{\mathcal{T}}\right)$ is a solution of the discrete problem (5), then

$$
\left|\left\langle\mathbf{R}\left(u_{\mathcal{T}}\right), v\right\rangle\right| \lesssim \sum_{T \in \mathcal{T}} \eta_{\mathcal{T}}\left(u_{\mathcal{T}} ; T\right)\|\nabla v\|_{\omega_{\mathcal{T}}(T)}
$$

for all $v \in H^{1}(\Omega)$. Moreover,

$$
\left\|\mathbf{R}\left(u_{\mathcal{T}}\right)\right\|_{\left(H^{1}(\Omega)\right)^{\prime}}:=\sup _{0 \neq v \in H^{1}(\Omega)} \frac{\left\langle\mathbf{R}\left(u_{\mathcal{T}}\right), v\right\rangle}{\|v\|_{H^{1}(\Omega)}} \lesssim \eta_{\mathcal{T}}\left(u_{\mathcal{T}}\right) .
$$

Definition 2.9 (Oscillation). For $v_{\mathcal{T}} \in \mathbb{V}_{\mathcal{T}}$ we define the local oscillation $\operatorname{osc}_{\mathcal{T}}\left(v_{\mathcal{T}} ; T\right)$ by

$$
\operatorname{osc}_{\mathcal{T}}^{2}\left(v_{\mathcal{T}} ; T\right):=h_{T}^{2}\|R-\bar{R}\|_{T}^{2}+h_{T}\|J-\bar{J}\|_{\partial T}^{2}, \quad \text { for all } T \in \mathcal{T},
$$

where $\left.\bar{R}\right|_{T}$ is the $L^{2}(T)$-projection of $R:=R\left(v_{\mathcal{T}}\right)$ onto $\mathcal{P}_{\ell}$, and for every side $S \subset$ $\partial T,\left.\bar{J}\right|_{S}$ is the $L^{2}(S)$-projection of $J:=J\left(v_{\mathcal{T}}\right)$ onto $\mathcal{P}_{\ell}$. The global oscillation $\operatorname{osc}_{\mathcal{T}}\left(v_{\mathcal{T}}\right)$ is given by

$$
\operatorname{osc}_{\mathcal{T}}^{2}\left(v_{\mathcal{T}}\right):=\sum_{T \in \mathcal{T}} \operatorname{osc}_{\mathcal{T}}^{2}\left(v_{\mathcal{T}} ; T\right)
$$

Whenever $\Xi$ is a subset of $\mathcal{T}, \operatorname{osc}_{\mathcal{T}}^{2}\left(v_{\mathcal{T}} ; \Xi\right)$ will denote the $\operatorname{sum} \sum_{T \in \Xi} \operatorname{osc}_{\mathcal{T}}^{2}\left(v_{\mathcal{T}} ; T\right)$.

The following result states that the estimator is bounded by the discrete solution locally and by a uniform constant independent of the mesh. This result is weaker than the usual lower bound, but sufficient to guarantee convergence, as first noticed by Siebert [21]. 
Theorem 2.10 (Estimator's Stability). For any $\mathcal{T} \in \mathbb{T}$, and $u_{\mathcal{T}} \in \tilde{E}_{\lambda}^{\mathcal{T}}$

$$
\eta_{\mathcal{T}}\left(u_{\mathcal{T}} ; T\right) \lesssim\left\|u_{\mathcal{T}}\right\|_{H^{1}\left(\omega_{\mathcal{T}}(T)\right)}, \quad \forall T \in \mathcal{T}, \quad \text { and } \quad \eta_{\mathcal{T}}\left(u_{\mathcal{T}} ; \mathcal{T}\right) \leq C_{\eta},
$$

where the uniform constant $C_{\eta}>0$ depends only on data and mesh regularity.

The proof of this theorem follows the steps of the usual proof of the lower bound through bubble functions, plus a bound for the oscillation terms, which is a consequence of the fact that, if $\mathcal{T} \in \mathbb{T}$ and $T \in \mathcal{T}$, then

$$
\operatorname{osc}_{\mathcal{T}}\left(v_{\mathcal{T}} ; T\right) \lesssim h_{T}\left(1+\Lambda\left(v_{\mathcal{T}}\right)\right)\left\|v_{\mathcal{T}}\right\|_{H^{1}\left(\omega_{\mathcal{T}}(T)\right)}, \quad \text { for all } v_{\mathcal{T}} \in \mathbb{V}_{\mathcal{T}} .
$$

This bound can be obtained as in [10], using the $W^{1, \infty}$ regularity of $\mathcal{A}$ and inverse inequalities.

\section{Convergence of a general adaptive loop}

This section is devoted to the proof of a plain convergence result (without rate) of adaptive algorithms using reasonable marking strategies. The result holds for simple as well as multiple eigenvalues, and the proof makes use of the new ideas presented in [21]. Specifically, we consider the following

Algorithm 1. Let $\mathcal{T}_{0}$ be any initial mesh, and let $k=0$.

1. $\left(\lambda_{k}, u_{k}\right):=\operatorname{SOLVE}\left(\mathbb{V}_{k}\right)$.

2. $\left\{\eta_{k}(T)\right\}_{T \in \mathcal{T}_{k}}:=\operatorname{ESTIMATE}\left(\lambda_{k}, u_{k}, \mathcal{T}_{k}\right)$.

3. $\mathcal{M}_{k}:=\operatorname{MARK}\left(\left\{\eta_{k}(T)\right\}_{T \in \mathcal{T}_{k}}, \mathcal{T}_{k}\right)$.

4. $\mathcal{T}_{k+1}:=\operatorname{REFINE}\left(\mathcal{T}_{k}, \mathcal{M}_{k}\right)$, increment $k$ and go to step 1.

We now describe the four main steps of the algorithm. Let $j_{0}$ be the index of the eigenvalue $\lambda$ of the continuous problem to be approximated. For a conforming triangulation $\mathcal{T}_{k}$ of $\Omega$, the module SOLVE takes the space $\mathbb{V}_{k}:=\mathbb{V}_{\mathcal{T}_{k}}$ as input argument and outputs the $j_{0}$-th eigenvalue of the discrete problem (5) with $\mathcal{T}=\mathcal{T}_{k}$, i.e., $\lambda_{k}:=\lambda_{j_{0}, \mathcal{T}_{k}}$, and a corresponding eigenfunction $u_{k} \in \mathbb{V}_{k}$. Therefore, $\lambda_{k}$ and $u_{k}$ satisfy

$$
\left\{\begin{array}{l}
a\left(u_{k}, v_{k}\right)=\lambda_{k} b\left(u_{k}, v_{k}\right), \quad \forall v_{k} \in \mathbb{V}_{k}, \\
\left\|u_{k}\right\|_{b}=1 .
\end{array}\right.
$$

Given $\mathcal{T}_{k}$ and the corresponding outputs $\lambda_{k}$ and $u_{k}$ of SOLVE, the module ESTIMATE computes and outputs the a posteriori error estimators $\left\{\eta_{k}(T)\right\}_{T \in \mathcal{T}_{k}}$, i.e.,

$$
\eta_{k}(T):=\eta_{\mathcal{T}_{k}}\left(u_{k} ; T\right) .
$$

Based upon the a posteriori error indicators $\left\{\eta_{k}(T)\right\}_{T \in \mathcal{T}_{k}}$, the module MARK collects elements of $\mathcal{T}_{k}$ in $\mathcal{M}_{k}$. The only requirement that we make on the module MARK is that the set of marked elements $\mathcal{M}_{k}$ contains at least one element of $\mathcal{T}_{k}$ holding the largest value of estimator. That is, there exists one element $T_{k}^{\max } \in \mathcal{M}_{k}$ such that

$$
\eta_{k}\left(T_{k}^{\max }\right)=\max _{T \in \mathcal{T}_{k}} \eta_{k}(T) .
$$


Whenever a marking strategy satisfies this assumption, we call it reasonable, since this is what practitioners do in order to maximize the error reduction with a minimum effort. It is easy to check that the most commonly used marking strategies, e.g., Maximum strategy, Equidistribution strategy, and the original Dörfler's strategy fulfill this condition.

The refinement procedure REFINE takes the triangulation $\mathcal{T}_{k}$ and the subset $\mathcal{M}_{k} \subset \mathcal{T}_{k}$ as input arguments. We require that all elements of $\mathcal{M}_{k}$ are refined at least $n$ times (with $n \geq 1$ ), by using the bisection procedure described in [23] to get a new conforming triangulation $\mathcal{T}_{k+1}$ of $\Omega$, which is a refinement of $\mathcal{T}_{k}$.

Therefore, starting with an initial triangulation $\mathcal{T}_{0}$, the iteration of the steps 1-4 generates a sequence $\left\{\mathcal{T}_{k}\right\}_{k \in \mathbb{N}_{0}} \subset \mathbb{T}$ and the corresponding outputs $\left(\lambda_{k}, u_{k}\right),\left\{\eta_{k}(T)\right\}_{T \in \mathcal{T}_{k}}, \mathcal{M}_{k}$ of the modules SOLVE, ESTIMATE and MARK, respectively.

The proof of convergence consists of essentially two steps. The first one is the proof that the sequence of discrete eigenpairs $\left\{\left(\lambda_{k}, u_{k}\right)\right\}_{k \in \mathbb{N}_{0}}$ contains a subsequence which converges to a limiting pair $\left(\lambda_{\infty}, u_{\infty}\right)$ in $\mathbb{R} \times H^{1}(\Omega)$; which is a consequence of the nestedness of the meshes (see Theorem 3.1 below). The second step consists in proving that the residual at $u_{\infty}$ is zero, and thus $\left(\lambda_{\infty}, u_{\infty}\right)$ is an eigenpair of the continuous problem in $H^{1}(\Omega)$, here the assumption of having a reasonable marking strategy plays an important role (see Theorems 3.7 and 3.8 below). Finally we prove that the whole sequence converges to $\tilde{E}_{\lambda_{\infty}}$, in the sense that $\lim _{k \rightarrow \infty} \operatorname{dist}_{H^{1}(\Omega)}\left(u_{k}, \tilde{E}_{\lambda_{\infty}}\right)=0$.

In order to start the proof, we now define the limiting space $\mathbb{V}_{\infty}:=\overline{\cup \mathbb{V}}_{k}{ }^{1}(\Omega)$, and note that $\mathbb{V}_{\infty}$ is thus a Hilbert space with the inner product inherited from $H^{1}(\Omega)$.

Theorem 3.1 (Convergence to a limiting pair). Let $\left\{\left(\lambda_{k}, u_{k}\right)\right\}_{k \in \mathbb{N}_{0}}$ be the sequence of computed eigenpairs given by Algorithm 1. There exist $\lambda_{\infty} \in \mathbb{R}$ and $u_{\infty} \in \mathbb{V}_{\infty}$ such that

$$
\left\{\begin{array}{l}
a\left(u_{\infty}, v\right)=\lambda_{\infty} b\left(u_{\infty}, v\right), \quad \forall v \in \mathbb{V}_{\infty}, \\
\left\|u_{\infty}\right\|_{b}=1 .
\end{array}\right.
$$

Moreover, $\lambda_{\infty}=\lim _{k \rightarrow \infty} \lambda_{k}$ and there exists a subsequence $\left\{u_{k_{m}}\right\}_{m \in \mathbb{N}_{0}}$ of $\left\{u_{k}\right\}_{k \in \mathbb{N}_{0}}$ such that

$$
u_{k_{m}} \longrightarrow u_{\infty} \text { in } H^{1}(\Omega) \text {. }
$$

Proof. Since $\mathcal{T}_{k+1}$ is always a refinement of $\mathcal{T}_{k}, \mathbb{V}_{k} \subset \mathbb{V}_{k+1}$, and by the MinimumMaximum principle $\left\{\lambda_{k}\right\}_{k \in \mathbb{N}_{0}}$ is a decreasing sequence bounded below by the $j_{0}$-th eigenvalue $\lambda$ of the continuous problem (2). Therefore, there exists $\lambda_{\infty}>0$ such that $\lambda_{k} \searrow \lambda_{\infty}$.

From (9) it follows that

$$
\left\|u_{k}\right\|_{a}^{2}=a\left(u_{k}, u_{k}\right)=\lambda_{k} b\left(u_{k}, u_{k}\right)=\lambda_{k}\left\|u_{k}\right\|_{b}^{2}=\lambda_{k} \rightarrow \lambda_{\infty},
$$

and therefore $\left\{u_{k}\right\}_{k \in \mathbb{N}_{0}}$ is a bounded sequence in $\mathbb{V}_{\infty}$. Then, there exists a subsequence $\left\{u_{k_{m}}\right\}_{m \in \mathbb{N}_{0}}$ weakly convergent in $\mathbb{V}_{\infty}$ to a function $u_{\infty} \in \mathbb{V}_{\infty}$, so

$$
u_{k_{m}} \longrightarrow u_{\infty} \quad \text { weakly in } H^{1}(\Omega) \text {. }
$$

Since the trace mapping is compact from $H^{1}(\Omega) \rightarrow L^{2}(\partial \Omega)$, we can extract a subsequence of the last one, which we still denote $\left\{u_{k_{m}}\right\}_{m \in \mathbb{N}_{0}}$, such that

$$
u_{k_{m}} \longrightarrow u_{\infty} \text { in } L^{2}(\partial \Omega) \text {. }
$$


We will now prove that $\left(\lambda_{\infty}, u_{\infty}\right)$ is an eigenpair in $\mathbb{V}_{\infty}$, i.e., (10) holds. Let $K \in \mathbb{N}_{0}$ and $v_{K} \in \mathbb{V}_{K}$. For $k_{m} \geq K$, since $\mathbb{V}_{K} \subset \mathbb{V}_{k_{m}}$ we have that $a\left(u_{k_{m}}, v_{K}\right)=\lambda_{k_{m}} b\left(u_{k_{m}}, v_{K}\right)$, and when $m$ tends to infinity, we obtain that $a\left(u_{\infty}, v_{K}\right)=\lambda_{\infty} b\left(u_{\infty}, v_{K}\right)$. Since $K \in \mathbb{N}_{0}$ and $v_{K} \in \mathbb{V}_{K}$ are arbitrary, by the density of $\bigcup_{k \in \mathbb{N}_{0}} \mathbb{V}_{k}$ in $\mathbb{V}_{\infty}$ we have that

$$
a\left(u_{\infty}, v\right)=\lambda_{\infty} b\left(u_{\infty}, v\right), \quad \forall v \in \mathbb{V}_{\infty}
$$

On the other hand, since $\left\|u_{k_{m}}\right\|_{b}=1$, considering (13) we conclude that $\left\|u_{\infty}\right\|_{b}=$ 1. Taking into account (14) we have that $\left\|u_{\infty}\right\|_{a}^{2}=\lambda_{\infty}\left\|u_{\infty}\right\|_{b}^{2}=\lambda_{\infty}$. Finally, from (11) it follows that $\left\|u_{k_{m}}\right\|_{a}^{2}=\lambda_{k_{m}} \longrightarrow \lambda_{\infty}=\left\|u_{\infty}\right\|_{a}$. This, together with (12) yields

$$
u_{k_{m}} \longrightarrow u_{\infty} \quad \text { (strongly) in } H^{1}(\Omega)
$$

Remark 3.2. It is important to notice that from any subsequence $\left\{\left(\lambda_{k_{m}}, u_{k_{m}}\right)\right\}_{m \in \mathbb{N}_{0}}$ of the original sequence $\left\{\left(\lambda_{k}, u_{k}\right)\right\}_{k \in \mathbb{N}_{0}}$, we can extract another subsequence $\left\{\left(\lambda_{k_{m_{p}}}, u_{k_{m_{p}}}\right)\right\}_{p \in \mathbb{N}_{0}}$, such that $u_{k_{m_{p}}}$ converges in $H^{1}(\Omega)$ to some function $\tilde{u}_{\infty} \in$ $\mathbb{V}_{\infty}$ that satisfies

$$
\left\{\begin{array}{l}
a\left(\tilde{u}_{\infty}, v\right)=\lambda_{\infty} b\left(\tilde{u}_{\infty}, v\right), \quad \forall v \in \mathbb{V}_{\infty}, \\
\left\|\tilde{u}_{\infty}\right\|_{b}=1 .
\end{array}\right.
$$

This fact will be used later on.

In order to prove that the residual $\mathbf{R}\left(u_{\infty}\right)$ vanishes, we will first prove that $\mathbf{R}\left(u_{k_{m}}\right) \rightarrow 0$ weakly. For this, we will classify the elements of each triangulation $\mathcal{T}_{k}$ in two sets. This classification was first used in [21] and is simpler than the one used in $[10,17]$.

Definition 3.3. Given the sequence $\left\{\mathcal{T}_{k}\right\}_{k \in \mathbb{N}_{0}}$ of triangulations, for each $k \in \mathbb{N}_{0}$ we define

$$
\mathcal{T}_{k}^{+}:=\left\{T \in \mathcal{T}_{k}: T \in \mathcal{T}_{m}, \quad \forall m \geq k\right\}, \quad \text { and } \quad \mathcal{T}_{k}^{0}:=\mathcal{T}_{k} \backslash \mathcal{T}_{k}^{+}
$$

Also, we consider

$$
\Omega_{k}^{+}:=\bigcup_{T \in \mathcal{T}_{k}^{+}} \omega_{k}(T), \quad \text { and } \quad \Omega_{k}^{0}:=\bigcup_{T \in \mathcal{T}_{k}^{0}} \omega_{k}(T),
$$

where $\omega_{k}(T):=\omega_{\mathcal{T}_{k}}(T)$. In words, $\mathcal{T}_{k}^{+}$is the set of elements of $\mathcal{T}_{k}$ that are not refined, and $\mathcal{T}_{k}^{0}$ consists of those elements which will eventually be refined. The region $\Omega_{k}^{+}$is the one covered by the unrefined elements and their inmediate neighbors, and $\Omega_{k}^{0}$ is the one covered by the elements which will be (eventually) refined later, and their neighbors.

We also need to introduce the following

Definition 3.4 (Meshsize function). We define $h_{k} \in L^{\infty}(\Omega)$ as the piecewise constant function

$$
\left.h_{k}\right|_{T}:=|T|^{1 / d}, \quad \forall T \in \mathcal{T}_{k}
$$


Since $\mathcal{T}_{k+1}$ is always a refinement of $\mathcal{T}_{k}$, for almost every $x \in \Omega$ there holds that $h_{k}(x)$ is monotonically decreasing and bounded from below by 0 . Therefore,

$$
h_{\infty}(x):=\lim _{k \rightarrow \infty} h_{k}(x)
$$

is well-defined for almost all $x \in \Omega$ and defines a function in $L^{\infty}(\Omega)$. Moreover, convergence is uniform, as is stated in the following lemma. (See Lemma 4.3 and Corollary 4.1 in [17]).

Lemma 3.5. The sequence $\left\{h_{k}\right\}_{k \in \mathbb{N}_{0}}$ converges to $h_{\infty}$ uniformly, i.e.,

$$
\lim _{k \rightarrow \infty}\left\|h_{k}-h_{\infty}\right\|_{L^{\infty}(\Omega)}=0,
$$

and if $\chi_{\Omega_{k}^{0}}$ denotes the characteristic function of $\Omega_{k}^{0}$ then

$$
\lim _{k \rightarrow \infty}\left\|h_{k} \chi_{\Omega_{k}^{0}}\right\|_{L^{\infty}(\Omega)}=0 .
$$

The proof of $\mathbf{R}\left(u_{\infty}\right)=0$ will exploit Siebert's idea [21] and is based on proving first convergence to zero of the estimators over the marked elements, and then the convergence of the residual $\mathbf{R}\left(u_{k_{m}}\right)$ to zero (weakly in $H^{-1}(\Omega)$ ).

Lemma 3.6 (Estimator on marked elements). Let $\left\{u_{k_{m}}\right\}_{m \in \mathbb{N}_{0}}$ be the convergent subsequence of $\left\{u_{k}\right\}_{k \in \mathbb{N}_{0}}$ given by Theorem 3.1. Then,

$$
\lim _{m \rightarrow \infty} \max _{T \in \mathcal{M}_{k_{m}}} \eta_{k_{m}}(T)=0 .
$$

Proof. In order not to clutter the notation, we still denote by $\left\{u_{k}\right\}_{k \in \mathbb{N}_{0}}$ the subsequence $\left\{u_{k_{m}}\right\}_{m \in \mathbb{N}_{0}}$, and by $\left\{\mathcal{T}_{k}\right\}_{k \in \mathbb{N}_{0}}$ the sequence $\left\{\mathcal{T}_{k_{m}}\right\}_{m \in \mathbb{N}_{0}}$. Let $T_{k} \in$ $\mathcal{M}_{k}$ be such that $\eta_{k}\left(T_{k}\right)=\max _{T \in \mathcal{M}_{k}} \eta_{k}(T)$. Using Theorem 2.10, we have that

$$
\eta_{k}\left(T_{k}\right) \lesssim\left\|u_{k}\right\|_{H^{1}\left(\omega_{k}\left(T_{k}\right)\right)} \leq\left\|u_{k}-u_{\infty}\right\|_{H^{1}(\Omega)}+\left\|u_{\infty}\right\|_{H^{1}\left(\omega_{k}\left(T_{k}\right)\right)} .
$$

Now, the first term in the right hand side of (15) tends to zero by Theorem 3.1, and since $T_{k} \in \mathcal{M}_{k} \subset \mathcal{T}_{k}^{0}$, by Lemma 3.5 we have

$$
\left|\omega_{k}\left(T_{k}\right)\right| \lesssim h_{T_{k}}^{d} \leq\left\|h_{k} \chi_{\Omega_{k}^{0}}\right\|_{L^{\infty}(\Omega)}^{d} \rightarrow 0, \quad \text { as } k \rightarrow \infty,
$$

and therefore, the second term in the right hand side of (15) also tends to zero.

Theorem 3.7 (Weak Convergence of the Residual). Let $\left\{u_{k_{m}}\right\}_{m \in \mathbb{N}_{0}}$ be the convergent subsequence of $\left\{u_{k}\right\}_{k \in \mathbb{N}_{0}}$ given by Theorem 3.1. Then,

$$
\lim _{m \rightarrow \infty}\left\langle\mathbf{R}\left(u_{k_{m}}\right), v\right\rangle=0, \quad \text { for all } v \in H^{1}(\Omega) .
$$

Proof. As in the last lemma, we denote by $\left\{u_{k}\right\}_{k \in \mathbb{N}_{0}}$ the subsequence $\left\{u_{k_{m}}\right\}_{m \in \mathbb{N}_{0}}$, and by $\left\{\mathcal{T}_{k}\right\}_{k \in \mathbb{N}_{0}}$ the sequence $\left\{\mathcal{T}_{k_{m}}\right\}_{m \in \mathbb{N}_{0}}$, and we prove the result for $v \in$ $H^{2}(\Omega)$. Let $n \in \mathbb{N}$ and $k>n$. By Definition 3.3 we have that $\mathcal{T}_{n}^{+} \subset \mathcal{T}_{k}^{+} \subset \mathcal{T}_{k}$. Let $v_{k} \in \mathbb{V}_{k}$ be the Lagrange's interpolant of $v$. Since $\left\langle\mathbf{R}\left(u_{k}\right), v_{k}\right\rangle=0$, using (8), and Cauchy-Schwartz's inequality we have that

$$
\begin{aligned}
\left|\left\langle\mathbf{R}\left(u_{k}\right), v\right\rangle\right| & =\left|\left\langle\mathbf{R}\left(u_{k}\right), v-v_{k}\right\rangle\right| \lesssim \sum_{T \in \mathcal{T}_{k}} \eta_{k}(T)\left\|v-v_{k}\right\|_{H^{1}\left(\omega_{k}(T)\right)} \\
& \leq \sum_{T \in \mathcal{T}_{n}^{+}} \eta_{k}(T)\left\|v-v_{k}\right\|_{H^{1}\left(\omega_{k}(T)\right)}+\sum_{T \in \mathcal{T}_{k} \backslash \mathcal{T}_{n}^{+}} \eta_{k}(T)\left\|v-v_{k}\right\|_{H^{1}\left(\omega_{k}(T)\right)} \\
& \leq \eta_{k}\left(\mathcal{T}_{n}^{+}\right)\left\|v-v_{k}\right\|_{H^{1}\left(\Omega_{n}^{+}\right)}+\eta_{k}\left(\mathcal{T}_{k} \backslash \mathcal{T}_{n}^{+}\right)\left\|v-v_{k}\right\|_{H^{1}\left(\Omega_{n}^{0}\right)} .
\end{aligned}
$$


Taking into account the Estimator's Stability (Theorem 2.10), we have that $\eta_{k}\left(\mathcal{T}_{k} \backslash \mathcal{T}_{n}^{+}\right) \leq \eta_{k}\left(\mathcal{T}_{k}\right) \leq C_{\eta}$, and therefore,

$$
\left|\left\langle\mathbf{R}\left(u_{k}\right), v\right\rangle\right| \lesssim\left(C \eta_{k}\left(\mathcal{T}_{n}^{+}\right)+C_{\eta}\left\|h_{n} \chi_{\Omega_{n}^{0}}\right\|_{L^{\infty}(\Omega)}\right)\|v\|_{H^{2}(\Omega)},
$$

due to interpolation estimates.

In order to prove that $\left\langle\mathbf{R}\left(u_{k}\right), v\right\rangle \rightarrow 0$ as $k \rightarrow \infty$ we now let $\epsilon>0$ be arbitrary. Due to Lemma 3.5, there exists $n \in \mathbb{N}$ such that

$$
C_{\eta}\left\|h_{n} \chi_{\Omega_{n}^{0}}\right\|_{L^{\infty}(\Omega)}<\epsilon .
$$

On the other hand, since $\mathcal{T}_{n}^{+} \subset \mathcal{T}_{k}^{+} \subset \mathcal{T}_{k}$ and the marking strategy is reasonable,

$$
\eta_{k}\left(\mathcal{T}_{n}^{+}\right) \leq\left(\# \mathcal{T}_{n}^{+}\right)^{1 / 2} \max _{T \in \mathcal{T}_{n}^{+}} \eta_{k}(T) \leq\left(\# \mathcal{T}_{n}^{+}\right)^{1 / 2} \max _{T \in \mathcal{M}_{k}} \eta_{k}(T) .
$$

Now, by Lemma 3.6, we can select $K>n$ such that $C \eta_{k}\left(\mathcal{T}_{n}^{+}\right)<\epsilon$, for all $k>K$.

Summarizing, we have proved that

$$
\lim _{k \rightarrow \infty}\left\langle\mathbf{R}\left(u_{k}\right), v\right\rangle=0, \quad \text { for all } v \in H^{2}(\Omega) .
$$

Since $H^{2}(\Omega)$ is dense in $H^{1}(\Omega)$, this limit is also zero for all $v \in H^{1}(\Omega)$.

As a consequence of the weak convergence of $\mathbf{R}\left(u_{k_{m}}\right)$ to zero, we now prove that $\left(\lambda_{\infty}, u_{\infty}\right)$ is an eigenpair of the continuous problem (2).

Theorem 3.8 (The limiting pair is an eigenpair). The limiting pair $\left(\lambda_{\infty}, u_{\infty}\right)$ of Theorem 3.1 is an eigenpair of the continuous problem (2). That is,

$$
\left\{\begin{array}{l}
a\left(u_{\infty}, v\right)=\lambda_{\infty} b\left(u_{\infty}, v\right), \quad \forall v \in H^{1}(\Omega), \\
\left\|u_{\infty}\right\|_{b}=1 .
\end{array}\right.
$$

Proof. We know that $\left\|u_{\infty}\right\|_{b}=1$ due to Theorem 3.1. It remains to prove that

$$
\left\langle\mathbf{R}\left(u_{\infty}\right), v\right\rangle=a\left(u_{\infty}, v\right)-\lambda_{\infty} b\left(u_{\infty}, v\right)=0, \quad \forall v \in H^{1}(\Omega) .
$$

If $v \in H^{1}(\Omega)$, and $\left\{u_{k_{m}}\right\}_{m \in \mathbb{N}_{0}}$ is the convergent subsequence of $\left\{u_{k}\right\}_{k \in \mathbb{N}_{0}}$ given by Theorem 3.1, then

$$
\begin{aligned}
\left|\left\langle\mathbf{R}\left(u_{\infty}\right), v\right\rangle\right| & =\left|\left\langle\mathbf{R}\left(u_{\infty}\right)-\mathbf{R}\left(u_{k_{m}}\right), v\right\rangle+\left\langle\mathbf{R}\left(u_{k_{m}}\right), v\right\rangle\right| \\
& \leq\left|a\left(u_{\infty}-u_{k_{m}}, v\right)\right|+\left|b\left(\lambda_{\infty} u_{\infty}-\lambda_{k_{m}} u_{k_{m}}, v\right)\right|+\left|\left\langle\mathbf{R}\left(u_{k_{m}}\right), v\right\rangle\right| \\
& \leq\left\|u_{\infty}-u_{k_{m}}\right\|_{a}\|v\|_{a}+\left\|\lambda_{\infty} u_{\infty}-\lambda_{k_{m}} u_{k_{m}}\right\|_{b}\|v\|_{b}+\left|\left\langle\mathbf{R}\left(u_{k_{m}}\right), v\right\rangle\right| .
\end{aligned}
$$

Since $\lambda_{k_{m}} \rightarrow \lambda_{\infty}$ and $u_{k_{m}} \rightarrow u_{\infty}$ (in $H^{1}(\Omega)$ and $L^{2}(\partial \Omega)$ ), Theorem 3.7 implies that the right hand side in the last inequality tends to zero when $m$ tends to infinity. Therefore,

$$
\left\langle\mathbf{R}\left(u_{\infty}\right), v\right\rangle=0, \quad \text { for all } v \in H^{1}(\Omega) .
$$

We now state and prove the main result of this section.

Theorem 3.9 (General Convergence Result 1). Let $\left\{\left(\lambda_{k}, u_{k}\right)\right\}_{k \in \mathbb{N}_{0}}$ denote the whole sequence of computed eigenpairs obtained with Algorithm 1. Then, there exists an eigenvalue $\lambda$ of the continuous problem (2) such that

$$
\lim _{k \rightarrow \infty} \lambda_{k}=\lambda \quad \text { and } \quad \lim _{k \rightarrow \infty} \operatorname{dist}_{H^{1}(\Omega)}\left(u_{k}, \tilde{E}_{\lambda}\right)=0 .
$$


Proof. By Theorem 3.1, taking $\lambda:=\lambda_{\infty}$, we have that $\lim _{k \rightarrow \infty} \lambda_{k}=\lambda$, and by Theorem 3.8, $\lambda$ is an eigenvalue of the continuous problem (2). In order to prove that $\lim _{k \rightarrow \infty} \operatorname{dist}_{H^{1}(\Omega)}\left(u_{k}, \tilde{E}_{\lambda}\right)=0$ we argue by contradiction. If the result were not true, there would exist a number $\epsilon>0$ and a subsequence $\left\{u_{k_{m}}\right\}_{m \in \mathbb{N}_{0}}$ of $\left\{u_{k}\right\}_{k \in \mathbb{N}_{0}}$ such that

$$
\operatorname{dist}_{H^{1}(\Omega)}\left(u_{k_{m}}, \tilde{E}_{\lambda}\right)>\epsilon, \quad \forall m \in \mathbb{N}_{0} .
$$

By Remark 3.2 it is possible to extract a subsequence of $\left\{u_{k_{m}}\right\}_{m \in \mathbb{N}_{0}}$ which still converges to some function $\tilde{u}_{\infty} \in \mathbb{V}_{\infty}$. By the arguments in the proof of Theorem $3.8, \tilde{u}_{\infty}$ is an eigenfunction of the continuous problem (2) corresponding to the same eigenvalue $\lambda$. That is, a subsequence of $\left\{u_{k_{m}}\right\}_{m \in \mathbb{N}_{0}}$ converges to an eigenfunction in $\tilde{E}_{\lambda}$, this contradicts (16) and completes the proof.

We have proved that the discrete eigenvalues converge to an eigenvalue of the continuous problem, and the discrete eigenfunctions converge to the set of the corresponding continuous eigenfunctions. But there is still an open question: Given that we have chosen $\lambda_{k}$ as the $j_{0}$-th eigenvalue of the discrete problem over $\mathcal{T}_{k}$, is it true that $\left\{\lambda_{k}\right\}_{k \in \mathbb{N}_{0}}$ converges to the $j_{0}$-th eigenvalue of the continuous problem? The answer is affirmative for a large number of problems, but not necessarily for all. There could be some pathological cases in which looking for the $j_{0}$-th eigenvalue we converge to one that is larger. We now state a sufficient assumption on problem (2) to guarantee that convergence holds to the desired eigenvalue/eigenfunction.

Assumption 3.1 (Non-Degeneracy Assumption). We will say that problem (2) satisfies the Non-Degeneracy Assumption if whenever $u$ is an eigenfunction of (2), there is no nonempty open subset $\mathcal{O}$ of $\Omega$ such that $\left.u\right|_{\mathcal{O}} \in \mathcal{P}_{\ell}(\mathcal{O})$.

Theorem 3.10 (General Convergence Result 2). Let us suppose that the continuous problem (2) satisfies the Non-Degeneracy Assumption 3.1, let $\left\{\left(\lambda_{k}, u_{k}\right)\right\}_{k \in \mathbb{N}_{0}}$ denote the whole sequence of computed eigenpairs obtained through Algorithm 1 , and let $\lambda$ denote the $j_{0}$-th eigenvalue of the continuous problem (2). Then,

$$
\lim _{k \rightarrow \infty} \lambda_{k}=\lambda \quad \text { and } \quad \lim _{k \rightarrow \infty} \operatorname{dist}_{H^{1}(\Omega)}\left(u_{k}, \tilde{E}_{\lambda}\right)=0
$$

Before embarking into the proof of this theorem, we state assumptions on the coefficients $\mathcal{A}$ and $c$ which guarantee non-degeneracy of the problem. The following lemma is a consequence of the results in [16], and the proof follows the lines of Lemma 6.1 in [10].

Lemma 3.11. If $\mathcal{A}$ is continuous, and piecewise $\mathcal{P}_{1}$, and c is piecewise constant, then problem (2) satisfies the Non-Degeneracy Assumption 3.1.

The next lemma states that under the non-degeneracy assumption, $h_{k} \rightarrow 0$ uniformly. It may seem confusing at first sight that this holds in general for adaptive meshes. However, after a second thought, it is a natural consequence of the convergence of the adaptive method, plus the nondegeneracy of the solution being approximated. It is also important to emphasize that the uniform convergence of $h_{k}$ to zero is not in contradiction with the spirit of adaptivity and nonlinear approximation results, since even though the convergence is uniform, the mesh-size function may have a strong grading around singularities. More 
precisely, uniform convergence of mesh-size does not imply quasi-uniformity of the meshes. This is already hinted by the explicit constructions of optimal meshes given in $[15,11]$.

Lemma 3.12. Let $\left\{h_{k}\right\}_{k \in \mathbb{N}_{0}}$ denote the sequence of meshsize functions obtained with Algorithm 1. If the continuous problem (2) satisfies the Non-Degeneracy Assumption 3.1, then $\left\|h_{k}\right\|_{L^{\infty}(\Omega)} \rightarrow 0$ as $k \rightarrow \infty$.

Proof. We argue by contradiction. If $\left\|h_{k}\right\|_{L^{\infty}(\Omega)}$ does not tend to zero, there exists $k_{0} \in \mathbb{N}_{0}$ and $T \in \mathcal{T}_{k_{0}}$ such that $T \in \mathcal{T}_{k}$, for all $k \geq k_{0}$. Let $\left\{u_{k_{m}}\right\}_{m \in \mathbb{N}_{0}}$ be the convergent subsequence of $\left\{u_{k}\right\}_{k \in \mathbb{N}_{0}}$ given by Theorem 3.1. Since $\| u_{k_{m}}-$ $u_{\infty} \|_{L^{2}(T)} \rightarrow 0$ as $m \rightarrow \infty$, and $\left.u_{k_{m}}\right|_{T} \in \mathcal{P}_{\ell}(T)$, for all $m \in \mathbb{N}_{0}$; using that $\mathcal{P}_{\ell}(T)$ is a finite dimensional space we conclude that

$$
\left.u_{\infty}\right|_{T} \in \mathcal{P}_{\ell}(T) .
$$

Theorem 3.8 states that $u_{\infty}$ is an eigenfunction of (2) and thus (17) contradicts the Non-Degeneracy Assumption 3.1.

It is important to notice that the convergence of $h_{k}$ to zero is not an assumption, but a consequence of the fact that a subsequence is converging to an eigenfunction $u_{\infty}$ and the Non-Degeneracy Assumption 3.1.

Proof of Theorem 3.10. In view of Theorem 3.9 it remains to prove that $\lambda_{k}$ converges to the $j_{0}$-th eigenvalue of (2). By Lemma 3.12 this follows from (6).

\section{Further error estimates}

Up to this point we have proved convergence (without rate) for any reasonable marking strategy. The next goal is to prove optimality. In this section we state some results that will be essential for proving optimality in the next section.

\subsection{A priori error estimates}

Recall that we consider the approximation of a certain fixed eigenvalue $\lambda=\lambda_{j_{0}}$ of the continuous problem (2), $j \in \mathbb{N}$ denotes the first index that satisfies $\lambda=\lambda_{j}$, and $R$ denotes its multiplicity.

If $u \in \tilde{E}_{\lambda}$ and $u_{\mathcal{T}} \in \tilde{E}_{\lambda}^{\mathcal{T}}$ then

$$
\begin{aligned}
a\left(u-u_{\mathcal{T}}, u-u_{\mathcal{T}}\right) & =a(u, u)+a\left(u_{\mathcal{T}}, u_{\mathcal{T}}\right)-2 a\left(u, u_{\mathcal{T}}\right)=\lambda+\Lambda\left(u_{\mathcal{T}}\right)-2 \lambda b\left(u, u_{\mathcal{T}}\right) \\
& =\Lambda\left(u_{\mathcal{T}}\right)-\lambda+\lambda\left(2-2 b\left(u, u_{\mathcal{T}}\right)\right)=\Lambda\left(u_{\mathcal{T}}\right)-\lambda+\lambda b\left(u-u_{\mathcal{T}}, u-u_{\mathcal{T}}\right),
\end{aligned}
$$

and thus,

$$
\left\|u-u_{\mathcal{T}}\right\|_{a}^{2}=\Lambda\left(u_{\mathcal{T}}\right)-\lambda+\lambda\left\|u-u_{\mathcal{T}}\right\|_{b}^{2} .
$$

As an immediate consequence of (18) we obtain the following

Theorem 4.1. If $u_{\mathcal{T}} \in \tilde{E}_{\lambda}^{\mathcal{T}}$, then $\Lambda\left(u_{\mathcal{T}}\right)-\lambda \leq \operatorname{dist}_{a}^{2}\left(u_{\mathcal{T}}, \tilde{E}_{\lambda}\right)$.

We will use the next auxiliary lemma in the proofs of several results. 
Lemma 4.2. Let $\mathcal{T} \in \mathbb{T}$. For all $u_{\mathcal{T}}, v_{\mathcal{T}} \in \mathbb{V}_{\mathcal{T}}$ with $\left\|u_{\mathcal{T}}\right\|_{b}=\left\|v_{\mathcal{T}}\right\|_{b}=1$, there holds

$$
\left\|\Lambda\left(u_{\mathcal{T}}\right) u_{\mathcal{T}}-\Lambda\left(v_{\mathcal{T}}\right) v_{\mathcal{T}}\right\|_{b} \leq\left(C_{T} \Lambda\left(u_{\mathcal{T}}\right)+\sqrt{\Lambda\left(u_{\mathcal{T}}\right)}+\sqrt{\Lambda\left(v_{\mathcal{T}}\right)}\right)\left\|u_{\mathcal{T}}-v_{\mathcal{T}}\right\|_{a}
$$

where $C_{T}$ is the constant from the trace inequality (1).

Proof. Let $\mathcal{T} \in \mathbb{T}$ and $u_{\mathcal{T}}, v_{\mathcal{T}} \in \mathbb{V}_{\mathcal{T}}$ with $\left\|u_{\mathcal{T}}\right\|_{b}=\left\|v_{\mathcal{T}}\right\|_{b}=1$. We have

$$
\begin{aligned}
\| \Lambda\left(u_{\mathcal{T}}\right) u_{\mathcal{T}} & -\Lambda\left(v_{\mathcal{T}}\right) v_{\mathcal{T}}\left\|_{b} \leq \Lambda\left(u_{\mathcal{T}}\right)\right\| u_{\mathcal{T}}-v_{\mathcal{T}}\left\|_{b}+\left|\Lambda\left(u_{\mathcal{T}}\right)-\Lambda\left(v_{\mathcal{T}}\right)\right|\right\| v_{\mathcal{T}} \|_{b} \\
& \leq \Lambda\left(u_{\mathcal{T}}\right)\left\|u_{\mathcal{T}}-v_{\mathcal{T}}\right\|_{b}+\left(\sqrt{\Lambda\left(u_{\mathcal{T}}\right)}+\sqrt{\Lambda\left(v_{\mathcal{T}}\right)}\right)\left|\sqrt{\Lambda\left(u_{\mathcal{T}}\right)}-\sqrt{\Lambda\left(v_{\mathcal{T}}\right)}\right| \\
& \leq \Lambda\left(u_{\mathcal{T}}\right)\left\|u_{\mathcal{T}}-v_{\mathcal{T}}\right\|_{b}+\left(\sqrt{\Lambda\left(u_{\mathcal{T}}\right)}+\sqrt{\Lambda\left(v_{\mathcal{T}}\right)}\right)\left\|u_{\mathcal{T}}-v_{\mathcal{T}}\right\|_{a} \\
& \leq\left(C_{T} \Lambda\left(u_{\mathcal{T}}\right)+\sqrt{\Lambda\left(u_{\mathcal{T}}\right)}+\sqrt{\Lambda\left(v_{\mathcal{T}}\right)}\right)\left\|u_{\mathcal{T}}-v_{\mathcal{T}}\right\|_{a},
\end{aligned}
$$

where in the last inequality we have used the bound (1).

The proof of the following result can be found in [24].

Lemma 4.3 (Separation of eigenvalues). There exist $\mathbf{h}_{\mathbf{0}}>0$ and a separation constant $\rho>0$ such that for every $\mathcal{T}, \mathcal{T}_{*} \in \mathbb{T}$ with $h_{\mathcal{T}} \leq \mathbf{h}_{\mathbf{0}}$, and $\mathcal{T}_{*}$ a refinement of $\mathcal{T}$,

$$
\frac{\lambda_{j}}{\left|\lambda_{i, \mathcal{T}}-\lambda_{j}\right|} \leq \rho, \quad \text { and } \quad \frac{\lambda_{\mathcal{T}_{*}}}{\left|\lambda_{i, \mathcal{T}}-\lambda_{\mathcal{T}_{*}}\right|} \leq \rho \quad \text { if } \quad i \neq j, j+1, \cdots, j+R-1,
$$

where $\lambda_{\mathcal{I}_{*}}$ denotes any of the discrete eigenvalues $\lambda_{j, \mathcal{T}_{*}}, \lambda_{j+1, \mathcal{T}_{*}}, \ldots, \lambda_{j+R-1, \mathcal{I}_{*}}$.

Remark 4.4. Hereafter, several statements hold under the assumption of $\mathcal{T}$ being sufficiently fine $\left(h_{\mathcal{T}}\right.$ small enough). However, under the Non-Degeneracy Assumption $3.1, h_{\mathcal{T}}$ will tend to zero as the adaptive algorithm iterates, and the fineness assumption will thus eventually hold. Let us also notice that all known optimality results are asymptotic, and so is ours. Despite the fact that estimates will hold starting at some iteration counter $k_{0}$, the algorithm converges starting from any initial mesh, and it does so at a quasi-optimal rate.

In order to prove the main a priori estimate of Theorem 4.8 we introduce now the elliptic projection operator and compare the error in the different norms.

Definition 4.5. Let $\mathcal{Q}_{\mathcal{T}}$ denote the elliptic projection of $H^{1}(\Omega)$ onto $\mathbb{V}_{\mathcal{T}}$; i.e., for each $v \in H^{1}(\Omega), \mathcal{Q}_{\mathcal{T}} v \in \mathbb{V}_{\mathcal{T}}$ satisfies $a\left(v-\mathcal{Q}_{\mathcal{T}} v, w_{\mathcal{T}}\right)=0$, for all $w_{\mathcal{T}} \in \mathbb{V}_{\mathcal{T}}$.

Lemma 4.6. If $u \in \tilde{E}_{\lambda}$ and $\left(\lambda_{i, \mathcal{T}}, u_{i, \mathcal{T}}\right)$ are solutions of $(5)$ for $i=1,2, \ldots, N_{\mathcal{T}}^{\partial}$, then,

$$
\left(\lambda_{i, \mathcal{T}}-\lambda\right) b\left(\mathcal{Q}_{\mathcal{T}} u, u_{i, \mathcal{T}}\right)=\lambda b\left(u-\mathcal{Q}_{\mathcal{T}} u, u_{i, \mathcal{T}}\right) .
$$

Proof. Since $a\left(\mathcal{Q}_{\mathcal{T}} u, u_{i, \mathcal{T}}\right)=a\left(u, u_{i, \mathcal{T}}\right)$ we have that $\lambda_{i, \mathcal{T}} b\left(\mathcal{Q}_{\mathcal{T}} u, u_{i, \mathcal{T}}\right)=\lambda b\left(u, u_{i, \mathcal{T}}\right)$. Subtracting $\lambda b\left(\mathcal{Q}_{\mathcal{T}} u, u_{i, \mathcal{T}}\right)$ from both sides, we obtain the claim of this lemma.

We recall that $r$ is a fixed number in $(0,1 / 2)$ so that the regularity estimate (4) holds for the solution of the Neumann problem. The following lemma can be proved with the usual duality trick of Aubin and Nitsche, and interpolation estimates in $H^{1+r}$. 
Lemma 4.7 (Aubin-Nitsche). There exists a constant $C_{A N}>0$ depending on the shape regularity of $\mathcal{T}_{0}$, and the constant appearing in (4), such that

$$
\left\|u-\mathcal{Q}_{\mathcal{T}} u\right\|_{b} \leq C_{A N} h_{\mathcal{T}}^{r}\left\|u-\mathcal{Q}_{\mathcal{T}} u\right\|_{a}, \quad \text { for all } u \in H^{1}(\Omega) \text { and all } \mathcal{T} \in \mathbb{T} .
$$

The proof of the following estimate is similar to the one presented in [13, 24] for the elliptic eigenvalue problem, but the fact that the discrete eigenfunctions do not generate all $\mathbb{V}_{\mathcal{T}}$ must be taken into account.

Theorem 4.8 (Main a priori estimate). Let $\mathbf{h}_{\mathbf{0}}$ and $\rho$ be as in Lemma 4.3. If $\lambda=\lambda_{j_{0}}$ is a simple eigenvalue, then

$$
\operatorname{dist}_{b}\left(u_{\mathcal{T}}, \tilde{E}_{\lambda}\right) \leq 2(1+\rho) C_{A N} h_{\mathcal{T}}^{r} \operatorname{dist}_{a}\left(u_{\mathcal{T}}, \tilde{E}_{\lambda}\right),
$$

for all $\mathcal{T} \in \mathbb{T}$ with $h_{\mathcal{T}} \leq \mathbf{h}_{\mathbf{0}}$, and $u_{\mathcal{T}} \in \tilde{E}_{\lambda}^{\mathcal{T}}$.

Proof. Assume that $\lambda=\lambda_{j_{0}}$ is a simple eigenvalue. Let $\mathcal{T} \in \mathbb{T}$ satisfy $h_{\mathcal{T}} \leq \mathbf{h}_{\mathbf{0}}$ and let $u_{\mathcal{T}} \in \tilde{E}_{\lambda}^{\mathcal{T}}$ be arbitrary. Let us take $u:=\tilde{\mathcal{P}}_{\lambda} u_{\mathcal{T}}$ and $\lambda_{\mathcal{T}}:=\Lambda\left(u_{\mathcal{T}}\right)$. Due to Remark 2.3 , it follows that $\beta:=b\left(\mathcal{Q}_{\mathcal{T}} u, u_{\mathcal{T}}\right)=\frac{1}{\lambda_{\mathcal{T}}} a\left(u, u_{\mathcal{T}}\right)$ is non-negative. Let $\left\{u_{i, \mathcal{T}} \mid i=1,2, \cdots, N_{\mathcal{T}}^{\partial}\right\}$ be a $b$-orthonormal subset of $\mathbb{V}_{\mathcal{T}}$, such that $\left(\lambda_{i, \mathcal{T}}, u_{i, \mathcal{T}}\right)$ are solutions of (5) for $i=1,2, \cdots, N_{\mathcal{T}}^{\partial}$, and $u_{j_{0}, \mathcal{T}}=u_{\mathcal{T}}$. Thus,

$$
\mathcal{Q}_{\mathcal{T}} u=\sum_{i=1}^{N_{\mathcal{T}}^{\partial}} b\left(\mathcal{Q}_{\mathcal{T}} u, u_{i, \mathcal{T}}\right) u_{i, \mathcal{T}}+v_{\mathcal{T}}
$$

where $v_{\mathcal{T}} \in \mathbb{V}_{\mathcal{T}}$ satifies $\left.v_{\mathcal{T}}\right|_{\partial \Omega} \equiv 0$, and then

$$
\left\|\mathcal{Q}_{\mathcal{T}} u-\beta u_{\mathcal{T}}\right\|_{b}^{2}=\sum_{\substack{i=1 \\ i \neq j_{0}}}^{N_{\mathcal{T}}^{\partial}} b\left(\mathcal{Q}_{\mathcal{T}} u, u_{i, \mathcal{T}}\right)^{2} .
$$

From Lemmas 4.6 and 4.3 and using that $h_{\mathcal{T}} \leq \mathbf{h}_{\mathbf{0}}$ it follows that

$$
\left\|\mathcal{Q}_{\mathcal{T}} u-\beta u_{\mathcal{T}}\right\|_{b}^{2}=\sum_{\substack{i=1 \\ i \neq j_{0}}}^{N_{\mathcal{T}}^{\partial}}\left(\frac{\lambda}{\lambda_{i, \mathcal{T}}-\lambda}\right)^{2} b\left(u-\mathcal{Q}_{\mathcal{T}} u, u_{i, \mathcal{T}}\right)^{2} \leq \rho^{2}\left\|u-\mathcal{Q}_{\mathcal{T}} u\right\|_{b}^{2} .
$$

By the triangle inequality $\left\|u-\beta u_{\mathcal{T}}\right\|_{b} \leq\left\|u-\mathcal{Q}_{\mathcal{T}} u\right\|_{b}+\left\|\mathcal{Q}_{\mathcal{T}} u-\beta u_{\mathcal{T}}\right\|_{b} \leq(1+$ $\rho)\left\|u-\mathcal{Q}_{\mathcal{T}} u\right\|_{b}$. Since $|\beta-1|=\left|\left\|\beta u_{\mathcal{T}}\right\|_{b}-\|u\|_{b}\right| \leq\left\|u-\beta u_{\mathcal{T}}\right\|_{b}$, we conclude that

$\left\|u-u_{\mathcal{T}}\right\|_{b} \leq\left\|u-\beta u_{\mathcal{T}}\right\|_{b}+\left\|(\beta-1) u_{\mathcal{T}}\right\|_{b} \leq 2\left\|u-\beta u_{\mathcal{T}}\right\|_{b} \leq 2(1+\rho)\left\|u-\mathcal{Q}_{\mathcal{T}} u\right\|_{b}$.

Finally, taking into account Lemma 4.7 and the definition of $\mathcal{Q}_{\mathcal{T}}$, we conclude

$$
\left\|u-u_{\mathcal{T}}\right\|_{b} \leq 2(1+\rho) C_{A N} h_{\mathcal{T}}^{r}\left\|u-\mathcal{Q}_{\mathcal{T}} u\right\|_{a} \leq 2(1+\rho) C_{A N} h_{\mathcal{T}}^{r}\left\|u-u_{\mathcal{T}}\right\|_{a} .
$$

The proof is complete observing that $u$ achieves $\operatorname{dist}_{b}\left(u_{\mathcal{T}}, \tilde{E}_{\lambda}\right)$ and $\operatorname{dist}_{a}\left(u_{\mathcal{T}}, \tilde{E}_{\lambda}\right)$.

Remark 4.9. Let $\mathbf{h}_{\mathbf{0}}$ and $\rho$ be as in Lemma 4.3 and let $\lambda$ be a simple eigenvalue. If $\mathcal{T}_{*}$ is a refinement of $\mathcal{T}$, the same proof of Theorem 4.8 allows us to conclude that if $h_{\mathcal{T}} \leq \mathbf{h}_{\mathbf{0}}$

$$
\operatorname{dist}_{b}\left(u_{\mathcal{T}}, \tilde{E}_{\lambda}^{\mathcal{T}_{*}}\right) \leq 2(1+\rho) C_{A N} h_{\mathcal{T}}^{r} \operatorname{dist}_{a}\left(u_{\mathcal{T}}, \tilde{E}_{\lambda}^{\mathcal{T}_{*}}\right), \quad \text { for any } u_{\mathcal{T}} \in \tilde{E}_{\lambda}^{\mathcal{T}} .
$$


In AFEM for symmetric elliptic problems the error is bound to decrease under mesh refinements. This result is false in general for eigenvalue problems due to their nonlinearity. We shall show however that the increase in the error can be controlled.

Theorem 4.10 (Error Stability). Let $\lambda$ be simple. Then, there exists a positive constant $\mathbf{h}_{\mathbf{1}} \leq \mathbf{h}_{\mathbf{0}}$ such that, if $\mathcal{T} \in \mathbb{T}$ with $h_{\mathcal{T}} \leq \mathbf{h}_{\mathbf{1}}$ and $\mathcal{T}_{*} \in \mathbb{T}$ is a refinement of $\mathcal{T}$,

$$
\operatorname{dist}_{a}\left(u_{\mathcal{T}_{*}}, \tilde{E}_{\lambda}\right) \lesssim \operatorname{dist}_{a}\left(u_{\mathcal{T}}, \tilde{E}_{\lambda}\right), \quad \text { for all } u_{\mathcal{T}} \in \tilde{E}_{\lambda}^{\mathcal{T}} \text { and } u_{\mathcal{T}_{*}} \in \tilde{E}_{\lambda}^{\mathcal{T}_{*}}
$$

Proof. Let $\mathcal{T}, \mathcal{T}_{*}$ be as in the assumptions, and let $u_{\mathcal{T}} \in \tilde{E}_{\lambda}^{\mathcal{T}}$ and $u_{\mathcal{T}_{*}} \in \tilde{E}_{\lambda}^{\mathcal{T}_{*}}$. By Theorem 4.8

$$
\operatorname{dist}_{b}\left(u_{\mathcal{T}_{*}}, \tilde{E}_{\lambda}\right) \lesssim h_{\mathcal{T}_{*}}^{r} \operatorname{dist}_{a}\left(u_{\mathcal{T}_{*}}, \tilde{E}_{\lambda}\right) .
$$

Now, if $\lambda_{\mathcal{T}}:=\Lambda\left(u_{\mathcal{T}}\right)$ and $\lambda_{\mathcal{T}_{*}}:=\Lambda\left(u_{\mathcal{T}_{*}}\right)$, using (18) for $\mathcal{T}_{*}$ instead of $\mathcal{T}$, we obtain

$$
\operatorname{dist}_{a}^{2}\left(u_{\mathcal{T}_{*}}, \tilde{E}_{\lambda}\right)=\lambda_{\mathcal{T}_{*}}-\lambda+\lambda \operatorname{dist}_{b}^{2}\left(u_{\mathcal{T}_{*}}, \tilde{E}_{\lambda}\right)
$$

due to the fact that the same $u \in \tilde{E}_{\lambda}$ achieves both $\operatorname{dist}_{a}\left(u_{\mathcal{T}_{*}}, \tilde{E}_{\lambda}\right)$ and $\operatorname{dist}_{b}\left(u_{\mathcal{T}_{*}}, \tilde{E}_{\lambda}\right)$ (see Remark 2.3). The fact that $\lambda_{\mathcal{T}_{*}} \leq \lambda_{\mathcal{T}}$, and Theorem 4.1 imply that

$$
\operatorname{dist}_{a}^{2}\left(u_{\mathcal{T}_{*}}, \tilde{E}_{\lambda}\right) \leq \lambda_{\mathcal{T}}-\lambda+\lambda \operatorname{dist}_{b}^{2}\left(u_{\mathcal{T}_{*}}, \tilde{E}_{\lambda}\right) \leq \operatorname{dist}_{a}^{2}\left(u_{\mathcal{T}}, \tilde{E}_{\lambda}\right)+\lambda \operatorname{dist}_{b}^{2}\left(u_{\mathcal{T}_{*}}, \tilde{E}_{\lambda}\right) .
$$

Considering (19), we can choose $\mathbf{h}_{\mathbf{1}}>0$ small enough such that the last term on the right-hand side can be absorbed on the left whenever $h_{\mathcal{T}_{*}} \leq \mathbf{h}_{\mathbf{1}}$.

\subsection{Further a posteriori error estimates}

In this section we will state some properties of the a posteriori error estimators that were not needed for proving convergence but will be used for proving quasioptimality. We start by stating an upper bound, which can be proved as in [9].

Lemma 4.11 (Upper bound). There exists a constant $C>0$, which only depends on the regularity of $\mathcal{T}_{0}$, such that for all eigenpairs $\left(\lambda_{\mathcal{T}}, u_{\mathcal{T}}\right)$ of the discrete problem (5),

$$
\operatorname{dist}_{a}\left(u_{\mathcal{T}}, \tilde{E}_{\lambda}\right) \leq C \eta_{\mathcal{T}}\left(u_{\mathcal{T}}\right)+\left(\frac{\lambda+\lambda_{\mathcal{T}}}{2}\right)^{1 / 2} \operatorname{dist}_{b}\left(u_{\mathcal{T}}, \tilde{E}_{\lambda}\right) .
$$

The following result is a quasi-localized upper bound for the distance between two nested solutions, and it is essential for proving optimality of adaptive FEM. Similar results were first used by Stevenson [22] and later on in [5, 7].

Lemma 4.12 (Quasi-localized Upper Bound). Let $\mathcal{T} \in \mathbb{T}, \mathcal{T}_{*} \in \mathbb{T}$ be a refinement of $\mathcal{T}$ and let $\mathcal{R}$ be the set of the elements in $\mathcal{T}$ which are refined to obtain $\mathcal{T}_{*}\left(\mathcal{R}=\mathcal{T} \backslash \mathcal{T}_{*}\right)$. Let $\left(\lambda_{\mathcal{T}}, u_{\mathcal{T}}\right)$ and $\left(\lambda_{\mathcal{T}_{*}}, u_{\mathcal{T}_{*}}\right)$ be solutions of the discrete problem (5) in $\mathcal{T}$ and $\mathcal{T}_{*}$, respectively. Then,

$$
\left\|u_{\mathcal{T}_{*}}-u_{\mathcal{T}}\right\|_{a} \lesssim \eta_{\mathcal{T}}\left(u_{\mathcal{T}} ; \mathcal{R}\right)+\left\|u_{\mathcal{T}_{*}}-u_{\mathcal{T}}\right\|_{b} .
$$


Proof. Let $\mathcal{T}, \mathcal{T}_{*}, \mathcal{R},\left(\lambda_{\mathcal{T}}, u_{\mathcal{T}}\right)$ and $\left(\lambda_{\mathcal{T}_{*}}, u_{\mathcal{T}_{*}}\right)$ be as in the assumptions, and let $e_{\mathcal{T}_{*}}:=u_{\mathcal{T}_{*}}-u_{\mathcal{T}}$. By simple calculations we have that

$$
\left\|e_{\mathcal{T}_{*}}\right\|_{a}^{2}=-\left\langle\mathbf{R}\left(u_{\mathcal{T}}\right), e_{\mathcal{T}_{*}}\right\rangle+\frac{\lambda_{\mathcal{T}_{*}}+\lambda_{\mathcal{T}}}{2}\left\|e_{\mathcal{T}_{*}}\right\|_{b}^{2} .
$$

Let $\Omega_{*}:=\bigcup_{T \in \mathcal{R}} T$ be the union of the refined elements, and $\mathbb{V}(\mathcal{R}):=\{v \in$ $\left.H^{1}\left(\Omega_{*}\right):\left.\quad v\right|_{T} \in \mathcal{P}_{\ell}(T), \quad \forall T \in \mathcal{R}\right\}$ the restriction of $\mathbb{V}_{\mathcal{T}}$ to $\Omega_{*}$. If $\mathcal{P}_{\mathcal{R}}:$ $H^{1}\left(\Omega_{*}\right) \rightarrow \mathbb{V}(\mathcal{R})$ denotes the Scott-Zhang interpolation operator [20], then

$$
\left\|e_{\mathcal{T}_{*}}-\mathcal{P}_{\mathcal{R}} e_{\mathcal{T}_{*}}\right\|_{T} \lesssim h_{T}\left\|\nabla e_{\mathcal{T}_{*}}\right\|_{\omega_{\mathcal{T}}(T)}, \quad \text { and } \quad\left\|e_{\mathcal{T}_{*}}-\mathcal{P}_{\mathcal{R}} e_{\mathcal{T}_{*}}\right\|_{\partial T} \lesssim h_{T}^{1 / 2}\left\|\nabla e_{\mathcal{T}_{*}}\right\|_{\omega_{\mathcal{T}}(T)},
$$

for all $T \in \mathcal{R}$. This operator is a projection and it preserves homogenous boundary values. Hence, it also preserves conforming boundary values, i.e., $\mathcal{P}_{\mathcal{R}} v=v$ on $\partial \Omega_{*}$ for $v \in H^{1}\left(\Omega_{*}\right)$ whenever $v=V$ on $\partial \Omega_{*}$ for some $V \in \mathbb{V}(\mathcal{R})$.

For the error $e_{\mathcal{T}_{*}} \in \mathbb{V}_{\mathcal{T}_{*}}$ we construct an approximation $e_{\mathcal{T}} \in \mathbb{V}_{\mathcal{T}}$ by

$$
e_{\mathcal{T}}:= \begin{cases}e_{\mathcal{T}_{*}} & \text { in } \Omega \backslash \Omega_{*} \\ \mathcal{P}_{\mathcal{R}} e_{\mathcal{T}_{*}} & \text { in } \Omega_{*} .\end{cases}
$$

Using (7) and taking into account that $e_{\mathcal{T}}=e_{\mathcal{T}_{*}}$ over all elements in $\mathcal{T} \backslash \mathcal{R}$, and the bounds (21), we obtain

$$
-\left\langle\mathbf{R}\left(u_{\mathcal{T}}\right), e_{\mathcal{T}_{*}}-e_{\mathcal{T}}\right\rangle \lesssim \sum_{T \in \mathcal{R}} \eta_{\mathcal{T}}\left(u_{\mathcal{T}} ; T\right)\left\|\nabla e_{\mathcal{T}_{*}}\right\|_{\omega_{\mathcal{T}}(T)} \lesssim \eta_{\mathcal{T}}\left(u_{\mathcal{T}} ; \mathcal{R}\right)\left\|e_{\mathcal{T}_{*}}\right\|_{a} .
$$

From (20) it follows that $\left\|e_{\mathcal{T}_{*}}\right\|_{a}^{2} \lesssim \eta_{\mathcal{T}}\left(u_{\mathcal{T}} ; \mathcal{R}\right)\left\|e_{\mathcal{T}_{*}}\right\|_{a}+\left\|e_{\mathcal{T}_{*}}\right\|_{b}^{2}$, and considering the trace inequality (1), this proof is complete.

The next result is the global lower bound for the error, whose proof follows the ideas given in [1].

Theorem 4.13 (Global Lower Bound). There exists a constant $C_{L}>0$ depending on the data such that, if $\mathcal{T} \in \mathbb{T}$, then

$$
C_{L} \eta_{\mathcal{T}}^{2}\left(u_{\mathcal{T}}\right) \leq \operatorname{dist}_{a}^{2}\left(u_{\mathcal{T}}, \tilde{E}_{\lambda}\right)+\operatorname{osc}_{\mathcal{T}}^{2}\left(u_{\mathcal{T}}\right), \quad \text { for any } \quad u_{\mathcal{T}} \in \tilde{E}_{\lambda}^{\mathcal{T}} .
$$

We finish this section with two theorems stating neat upper bounds (global and localized) for meshes that are sufficiently fine and simple eigenvalues. We show that the higher order terms in Lemmas 4.11 and 4.12 can be absorbed into the left using the main a priori estimate (see Theorem 4.8 and Remark 4.9).

Theorem 4.14 (Global Upper Bound). Assume that $\lambda=\lambda_{j_{0}}$ is simple. Then, there exist a constant $C_{U}>0$ depending on the data and a positive constant $\mathbf{h}_{\mathbf{2}} \leq \mathbf{h}_{\mathbf{0}}$ such that, if $\mathcal{T} \in \mathbb{T}$ with $h_{\mathcal{T}} \leq \mathbf{h}_{\mathbf{2}}$, then

$$
\operatorname{dist}_{a}^{2}\left(u_{\mathcal{T}}, \tilde{E}_{\lambda}\right) \leq C_{U} \eta_{\mathcal{T}}^{2}\left(u_{\mathcal{T}}\right), \quad \text { for any } \quad u_{\mathcal{T}} \in \tilde{E}_{\lambda}^{\mathcal{T}} \text {. }
$$

Proof. Let $\mathcal{T} \in \mathbb{T}$ and $u_{\mathcal{T}} \in \tilde{E}_{\lambda}^{\mathcal{T}}$. From Lemma 4.11 and Theorem 4.8 it follows that

$$
\operatorname{dist}_{a}\left(u_{\mathcal{T}}, \tilde{E}_{\lambda}\right) \lesssim \eta_{\mathcal{T}}\left(u_{\mathcal{T}}\right)+h_{\mathcal{T}}^{r} \operatorname{dist}_{a}\left(u_{\mathcal{T}}, \tilde{E}_{\lambda}\right),
$$

for $h_{\mathcal{T}} \leq \mathbf{h}_{\mathbf{0}}$. The claim of this theorem follows by noticing that we can select $\mathbf{h}_{\mathbf{2}} \leq \mathbf{h}_{\mathbf{0}}$ depending on the data small enough such that the second term on the right-hand side can be absorbed into the left if $h_{\mathcal{T}} \leq \mathbf{h}_{\mathbf{2}}$. 
Theorem 4.15 (Localized Upper Bound). Assume that $\lambda=\lambda_{j_{0}}$ is simple. Let $\mathcal{T}_{*} \in \mathbb{T}$ be a refinement of $\mathcal{T} \in \mathbb{T}$ and let $\mathcal{R}$ be the set of the elements in $\mathcal{T}$ which are refined to obtain $\mathcal{T}_{*}$, i.e., $\mathcal{R}=\mathcal{T} \backslash \mathcal{T}_{*}$. There exist a constant $C_{L U}>0$ depending on the data and $0<\mathbf{h}_{\mathbf{3}} \leq \mathbf{h}_{\mathbf{0}}$ such that, if $h_{\mathcal{T}} \leq \mathbf{h}_{\mathbf{3}}$,

$$
\operatorname{dist}_{a}^{2}\left(u_{\mathcal{T}}, \tilde{E}_{\lambda}^{\mathcal{T}_{*}}\right) \leq C_{L U} \eta_{\mathcal{T}}^{2}\left(u_{\mathcal{T}} ; \mathcal{R}\right), \quad \text { for any } \quad u_{\mathcal{T}} \in \tilde{E}_{\lambda}^{\mathcal{T}} .
$$

Proof. Let $\mathcal{T}, \mathcal{T}_{*}$, and $\mathcal{R}$ be as in the assumptions, and let $u_{\mathcal{T}} \in \tilde{E}_{\lambda}^{\mathcal{T}}$. From Lemma 4.12 and Remark 4.9 it follows that

$$
\operatorname{dist}_{a}\left(u_{\mathcal{T}}, \tilde{E}_{\lambda}^{\mathcal{T}_{*}}\right) \lesssim \eta_{\mathcal{T}}\left(u_{\mathcal{T}} ; \mathcal{R}\right)+h_{\mathcal{T}}^{r} \operatorname{dist}_{a}\left(u_{\mathcal{T}}, \tilde{E}_{\lambda}^{\mathcal{T}_{*}}\right),
$$

for $h_{\mathcal{T}} \leq \mathbf{h}_{\mathbf{0}}$. As in the last proof, we can select $\mathbf{h}_{\mathbf{3}}>0$ (depending on data) small enough in order to absorbe the second term in the right-hand side into the left if $h_{\mathcal{T}} \leq \mathbf{h}_{\mathbf{3}}$.

\subsection{Estimator and oscillation reductions}

In this section we study what happens with the estimator and the oscillation indicator when refining. We start observing that if $\mathcal{T} \in \mathbb{T}$ and $v_{\mathcal{T}}, w_{\mathcal{T}} \in \mathbb{V}_{\mathcal{T}}$, then

$$
\begin{aligned}
\eta_{\mathcal{T}}\left(w_{\mathcal{T}} ; T\right)= & \left(h_{T}^{2}\left\|R\left(w_{\mathcal{T}}\right)\right\|_{T}^{2}+h_{T}\left\|J\left(w_{\mathcal{T}}\right)\right\|_{\partial T}^{2}\right)^{1 / 2} \\
\leq & \left(h_{T}^{2}\left\|R\left(v_{\mathcal{T}}\right)\right\|_{T}^{2}+h_{T}\left\|J\left(v_{\mathcal{T}}\right)\right\|_{\partial T}^{2}\right)^{1 / 2} \\
& \quad+\left(\left(h_{T}\left\|R\left(v_{\mathcal{T}}\right)-R\left(w_{\mathcal{T}}\right)\right\|_{T}\right)^{2}+\left(h_{T}^{1 / 2}\left\|J\left(v_{\mathcal{T}}\right)-J\left(w_{\mathcal{T}}\right)\right\|_{\partial T}\right)^{2}\right)^{1 / 2} \\
\leq & \eta_{\mathcal{T}}\left(v_{\mathcal{T}} ; T\right)+h_{T}\left\|R\left(v_{\mathcal{T}}\right)-R\left(w_{\mathcal{T}}\right)\right\|_{T}+h_{T}^{1 / 2}\left\|J\left(v_{\mathcal{T}}\right)-J\left(w_{\mathcal{T}}\right)\right\|_{\partial T}
\end{aligned}
$$

After defining $g_{\mathcal{T}}\left(v_{\mathcal{T}}, w_{\mathcal{T}} ; T\right):=h_{T}\left\|R\left(v_{\mathcal{T}}\right)-R\left(w_{\mathcal{T}}\right)\right\|_{T}+h_{T}^{1 / 2}\left\|J\left(v_{\mathcal{T}}\right)-J\left(w_{\mathcal{T}}\right)\right\|_{\partial T}$, the last inequality reads

$$
\eta_{\mathcal{T}}\left(w_{\mathcal{T}} ; T\right) \leq \eta_{\mathcal{T}}\left(v_{\mathcal{T}} ; T\right)+g_{\mathcal{T}}\left(v_{\mathcal{T}}, w_{\mathcal{T}} ; T\right)
$$

and analogously we obtain for all $T \in \mathcal{T}$, and $v_{\mathcal{T}}, w_{\mathcal{T}} \in \mathbb{V}_{\mathcal{T}}$,

$$
\operatorname{osc}_{\mathcal{T}}\left(w_{\mathcal{T}} ; T\right) \leq \operatorname{osc}_{\mathcal{T}}\left(v_{\mathcal{T}} ; T\right)+g_{\mathcal{T}}\left(v_{\mathcal{T}}, w_{\mathcal{T}} ; T\right) .
$$

The quantity $g_{\mathcal{T}}$ thus accounts for the difference between the error indicators and oscillation terms of two discrete functions, and by a scaled trace theorem and an inverse inequality, it can be bounded by

$g_{\mathcal{T}}\left(v_{\mathcal{T}}, w_{\mathcal{T}} ; T\right) \lesssim\left\|v_{\mathcal{T}}-w_{\mathcal{T}}\right\|_{H^{1}\left(\omega_{\mathcal{T}}(T)\right)}+\left\|\Lambda\left(v_{\mathcal{T}}\right) v_{\mathcal{T}}-\Lambda\left(w_{\mathcal{T}}\right) w_{\mathcal{T}}\right\|_{\partial \Omega \cap \partial T}, \quad$ for all $T \in \mathcal{T}$.

After squaring and adding over all $T \in \mathcal{T}$, we also obtain

$$
\sum_{T \in \mathcal{T}} g_{\mathcal{T}}^{2}\left(v_{\mathcal{T}}, w_{\mathcal{T}} ; T\right) \lesssim\left\|v_{\mathcal{T}}-w_{\mathcal{T}}\right\|_{a}^{2}+\left\|\Lambda\left(v_{\mathcal{T}}\right) v_{\mathcal{T}}-\Lambda\left(w_{\mathcal{T}}\right) w_{\mathcal{T}}\right\|_{b}^{2}
$$

The next result quantifies the reduction of the estimator due to refinement, and its proof makes use of Young's inequality:

$$
a^{2} \leq(1+\delta) b^{2}+\left(1+\delta^{-1}\right) c^{2}, \quad \text { if } 0 \leq a \leq b+c, \quad \text { for all } \delta>0 .
$$


Proposition 4.16 (Estimator reduction). Let $\mathcal{T} \in \mathbb{T}$ and let $\mathcal{M}_{\mathcal{T}}$ be any subset of $\mathcal{T}$. Let $\mathcal{T}_{*} \in \mathbb{T}$ be obtained from $\mathcal{T}$ refining at least $n \geq 1$ times the elements in $\mathcal{M}_{\mathcal{T}}$ and let $c:=1-2^{-n / d}>0$. Then, there exists a constant $C_{E}>0$ depending on data such that if $u_{\mathcal{T}} \in \tilde{E}_{\lambda}^{\mathcal{T}}$ and $u_{\mathcal{T}_{*}} \in \tilde{E}_{\lambda}^{\mathcal{T}_{*}}$

$\eta_{\mathcal{T}_{*}}^{2}\left(u_{\mathcal{T}_{*}}\right) \leq(1+\delta)\left\{\eta_{\mathcal{T}}^{2}\left(u_{\mathcal{T}}\right)-c \eta_{\mathcal{T}}^{2}\left(u_{\mathcal{T}} ; \mathcal{M}_{\mathcal{T}}\right)\right\}+\left(1+\delta^{-1}\right) C_{E}\left\|u_{\mathcal{T}_{*}}-u_{\mathcal{T}}\right\|_{a}^{2}, \quad$ for all $\delta>0$.

Proof. Let $\mathcal{T}, \mathcal{M}_{\mathcal{T}}$ and $\mathcal{T}_{*}$ be as in the assumptions and let $u_{\mathcal{T}} \in \tilde{E}_{\lambda}^{\mathcal{T}}$ and $u_{\mathcal{T}_{*}} \in \tilde{E}_{\lambda}^{\mathcal{T}_{*}}$ be arbitrary. Applying Young's inequality with parameter $\delta$ to (22) we derive

$$
\eta_{\mathcal{T}_{*}}^{2}\left(u_{\mathcal{T}_{*}} ; T\right) \leq(1+\delta) \eta_{\mathcal{T}_{*}}^{2}\left(u_{\mathcal{T}} ; T\right)+\left(1+\delta^{-1}\right) g_{\mathcal{T}_{*}}^{2}\left(u_{\mathcal{T}}, u_{\mathcal{T}_{*}} ; T\right),
$$

for $T \in \mathcal{T}_{*}$. Summing over all elements $T \in \mathcal{T}_{*}$, and using (24) we have that

$$
\eta_{\mathcal{T}_{*}}^{2}\left(u_{\mathcal{T}_{*}}\right) \leq(1+\delta) \eta_{\mathcal{T}_{*}}^{2}\left(u_{\mathcal{T}}\right)+\left(1+\delta^{-1}\right) C_{E}\left\|u_{\mathcal{T}_{*}}-u_{\mathcal{T}}\right\|_{a}^{2}
$$

for some constant $C_{E}>0$, where we also have taken into account Lemma 4.2 to bound

$$
\left\|\Lambda\left(u_{\mathcal{T}}\right) u_{\mathcal{T}}-\Lambda\left(u_{\mathcal{T}_{*}}\right) u_{\mathcal{T}_{*}}\right\|_{b} \leq\left(C_{T} \lambda_{j+R-1, \mathcal{T}_{0}}+2 \sqrt{\lambda_{j+R-1, \mathcal{T}_{0}}}\right)\left\|u_{\mathcal{T}}-u_{\mathcal{T}_{*}}\right\|_{a} .
$$

Next, for an element $T \in \mathcal{T}$, we set $\mathcal{T}_{*, T}:=\left\{T^{\prime} \in \mathcal{T}_{*}: T^{\prime} \subset T\right\}$ and we note that $J\left(u_{\mathcal{T}}\right)=0$ on the sides of $\mathcal{T}_{*, T}$ that lie in the interior of $T$, since $u_{\mathcal{T}} \in \mathbb{V}_{\mathcal{T}}$. For $T \in \mathcal{M}_{\mathcal{T}}$ we obtain

$$
\begin{aligned}
\sum_{T^{\prime} \in \mathcal{T}_{*, T}} \eta_{\mathcal{T}_{*}}^{2}\left(u_{\mathcal{T}} ; T^{\prime}\right) & =\sum_{T^{\prime} \in \mathcal{T}_{*, T}}\left(h_{T^{\prime}}^{2}\left\|R\left(u_{\mathcal{T}}\right)\right\|_{T^{\prime}}^{2}+h_{T^{\prime}}\left\|J\left(u_{\mathcal{T}}\right)\right\|_{\partial T^{\prime}}^{2}\right) \\
& \leq 2^{-\frac{n}{d} 2} h_{T}^{2}\left\|R\left(u_{\mathcal{T}}\right)\right\|_{T}^{2}+2^{-\frac{n}{d}} h_{T}\left\|J\left(u_{\mathcal{T}}\right)\right\|_{\partial T}^{2} \leq 2^{-\frac{n}{d}} \eta_{\mathcal{T}}^{2}\left(u_{\mathcal{T}} ; T\right),
\end{aligned}
$$

since refinement by bisection implies $h_{T^{\prime}}=\left|T^{\prime}\right|^{\frac{1}{d}} \leq\left(2^{-n}|T|\right)^{\frac{1}{d}}=2^{-\frac{n}{d}} h_{T}$, for all $T^{\prime} \in \mathcal{T}_{*, T}$.

Now, the last estimation for $T \in \mathcal{M}_{\mathcal{T}}$ and the fact that $\sum_{T^{\prime} \in \mathcal{T}_{*, T}} \eta_{\mathcal{T}_{*}}^{2}\left(u_{\mathcal{T}} ; T^{\prime}\right) \leq$ $\eta_{\mathcal{T}}^{2}\left(u_{\mathcal{T}} ; T\right)$ for $T \in \mathcal{T} \backslash \mathcal{M}_{\mathcal{T}}$ imply that

$$
\begin{aligned}
\eta_{\mathcal{T}_{*}}^{2}\left(u_{\mathcal{T}}\right) & =\sum_{T \in \mathcal{T} \backslash \mathcal{M}_{\mathcal{T}}} \sum_{T^{\prime} \in \mathcal{T}_{*, T}} \eta_{\mathcal{T}_{*}}^{2}\left(u_{\mathcal{T}} ; T^{\prime}\right)+\sum_{T \in \mathcal{M}_{\mathcal{T}}} \sum_{T^{\prime} \in \mathcal{T}_{*}, T} \eta_{\mathcal{T}_{*}}^{2}\left(u_{\mathcal{T}} ; T^{\prime}\right) \\
& \leq \sum_{T \in \mathcal{T} \backslash \mathcal{M}_{\mathcal{T}}} \eta_{\mathcal{T}}^{2}\left(u_{\mathcal{T}} ; T\right)+\sum_{T \in \mathcal{M}_{\mathcal{T}}} 2^{-\frac{n}{d}} \eta_{\mathcal{T}}^{2}\left(u_{\mathcal{T}} ; T\right) \\
& =\eta_{\mathcal{T}}^{2}\left(u_{\mathcal{T}}\right)-\left(1-2^{-\frac{n}{d}}\right) \eta_{\mathcal{T}}^{2}\left(u_{\mathcal{T}} ; \mathcal{M}_{\mathcal{T}}\right) .
\end{aligned}
$$

Finally, inserting this bound in (25) the claim of this proposition follows.

The following result quantifies the change of the oscillation term due to refinement.

Proposition 4.17 (Perturbation of oscillation). Let $\mathcal{T} \in \mathbb{T}$. If $\mathcal{T}_{*} \in \mathbb{T}$ is a refinement of $\mathcal{T}$,

$\operatorname{osc}_{\mathcal{T}}^{2}\left(u_{\mathcal{T}} ; \mathcal{T} \cap \mathcal{T}_{*}\right) \leq 2 \operatorname{osc}_{\mathcal{T}_{*}}^{2}\left(u_{\mathcal{T}_{*}} ; \mathcal{T} \cap \mathcal{T}_{*}\right)+2 C_{E}\left\|u_{\mathcal{T}_{*}}-u_{\mathcal{T}}\right\|_{a}^{2}, \quad$ for all $u_{\mathcal{T}} \in \tilde{E}_{\lambda}^{\mathcal{T}}, u_{\mathcal{T}_{*}} \in \tilde{E}_{\lambda}^{\mathcal{T}_{*}}$. 
Proof. Let $\mathcal{T} \in \mathbb{T}$ and $\mathcal{T}_{*} \in \mathbb{T}$ be a refinement of $\mathcal{T}$. Let $u_{\mathcal{T}} \in \tilde{E}_{\lambda}^{\mathcal{T}}$ and $u_{\mathcal{T}_{*}} \in \tilde{E}_{\lambda}^{\mathcal{T}_{*}}$ be arbitrary. Applying Young's inequality to (23) we derive

$$
\operatorname{OSc}_{\mathcal{T}_{*}}^{2}\left(u_{\mathcal{T}} ; T\right) \leq 2 \operatorname{osc}_{\mathcal{T}_{*}}^{2}\left(u_{\mathcal{T}_{*}} ; T\right)+2 g_{\mathcal{T}_{*}}^{2}\left(u_{\mathcal{T}}, u_{\mathcal{T}_{*}} ; T\right)
$$

for all $T \in \mathcal{T} \cap \mathcal{T}_{*}$. Using that $\operatorname{osc}_{\mathcal{T}}\left(u_{\mathcal{T}} ; T\right)=\operatorname{osc}_{\mathcal{T}_{*}}\left(u_{\mathcal{T}} ; T\right)$, summing over all elements $T \in \mathcal{T} \cap \mathcal{T}_{*}$, using (24) and Lemma 4.2 again, we obtain the claim.

The next result is a consequence of the estimation (23) and the bound (24), and it will be used in the next section in order to prove the optimality of the approximations (see Lemma 5.3 below).

Lemma 4.18. If $\mathcal{T} \in \mathbb{T}$ and $\left(\lambda_{\mathcal{T}}, u_{\mathcal{T}}\right)$ is a solution of the discrete problem (5), then

$$
\operatorname{osc}_{\mathcal{T}}^{2}\left(u_{\mathcal{T}}\right) \lesssim \operatorname{osc}_{\mathcal{T}}^{2}\left(v_{\mathcal{T}}\right)+\left\|u_{\mathcal{T}}-v_{\mathcal{T}}\right\|_{a}^{2}, \quad \text { for all } v_{\mathcal{T}} \in \mathbb{V}_{\mathcal{T}} \text { with }\left\|v_{\mathcal{T}}\right\|_{b}=1 .
$$

Proof. Let $\mathcal{T} \in \mathbb{T}$ and $\left(\lambda_{\mathcal{T}}, u_{\mathcal{T}}\right)$ be a solution of the discrete problem (5). Let $v_{\mathcal{T}} \in \mathbb{V}_{\mathcal{T}}$ be such that $\left\|v_{\mathcal{T}}\right\|_{b}=1$.

1 We assume first that $a\left(u_{\mathcal{T}}, v_{\mathcal{T}}\right) \geq 0$. From (23), it follows that

$$
\operatorname{osc}_{\mathcal{T}}^{2}\left(u_{\mathcal{T}} ; T\right) \leq 2\left(\operatorname{osc}_{\mathcal{T}}^{2}\left(v_{\mathcal{T}} ; T\right)+g_{\mathcal{T}}^{2}\left(v_{\mathcal{T}}, u_{\mathcal{T}} ; T\right)\right), \quad \text { for all } T \in \mathcal{T} .
$$

After adding over all the elements $T \in \mathcal{T}$, by (24) we obtain

$$
\operatorname{osc}_{\mathcal{T}}^{2}\left(u_{\mathcal{T}}\right) \lesssim \operatorname{osc}_{\mathcal{T}}^{2}\left(v_{\mathcal{T}}\right)+\left\|u_{\mathcal{T}}-v_{\mathcal{T}}\right\|_{a}^{2}+\left\|\lambda_{\mathcal{T}} u_{\mathcal{T}}-\Lambda\left(v_{\mathcal{T}}\right) v_{\mathcal{T}}\right\|_{b}^{2}
$$

Therefore, it is sufficient to prove that $\left\|\lambda_{\mathcal{T}} u_{\mathcal{T}}-\Lambda\left(v_{\mathcal{T}}\right) v_{\mathcal{T}}\right\|_{b}^{2} \lesssim\left\|u_{\mathcal{T}}-v_{\mathcal{T}}\right\|_{a}^{2}$. On the one hand, if $\Lambda\left(v_{\mathcal{T}}\right) \geq 1$, we have that

$$
\begin{aligned}
\left\|\lambda_{\mathcal{T}} u_{\mathcal{T}}-\Lambda\left(v_{\mathcal{T}}\right) v_{\mathcal{T}}\right\|_{b}^{2} & =\lambda_{\mathcal{T}}+\Lambda\left(v_{\mathcal{T}}\right)-2 \lambda_{\mathcal{T}} \Lambda\left(v_{\mathcal{T}}\right) b\left(u_{\mathcal{T}}, v_{\mathcal{T}}\right) \\
& =\lambda_{\mathcal{T}}+\Lambda\left(v_{\mathcal{T}}\right)-2 \Lambda\left(v_{\mathcal{T}}\right) a\left(u_{\mathcal{T}}, v_{\mathcal{T}}\right) \\
& \leq \lambda_{\mathcal{T}}+\Lambda\left(v_{\mathcal{T}}\right)-2 a\left(u_{\mathcal{T}}, v_{\mathcal{T}}\right)=\left\|u_{\mathcal{T}}-v_{\mathcal{T}}\right\|_{a}^{2}
\end{aligned}
$$

On the other hand, if $\Lambda\left(v_{\mathcal{T}}\right) \leq 1$, Lemma 4.2 implies

$\left\|\lambda_{\mathcal{T}} u_{\mathcal{T}}-\Lambda\left(v_{\mathcal{T}}\right) v_{\mathcal{T}}\right\|_{b} \leq\left(C_{T} \lambda_{\mathcal{T}}+\sqrt{\lambda_{\mathcal{T}}}+\sqrt{\Lambda\left(v_{\mathcal{T}}\right)}\right)\left\|u_{\mathcal{T}}-v_{\mathcal{T}}\right\|_{a} \lesssim\left\|u_{\mathcal{T}}-v_{\mathcal{T}}\right\|_{a}$.

2 If $a\left(u_{\mathcal{T}}, v_{\mathcal{T}}\right)<0$, using the first part we have that

$$
\operatorname{osc}_{\mathcal{T}}^{2}\left(u_{\mathcal{T}}\right) \lesssim \operatorname{osc}_{\mathcal{T}}^{2}\left(-v_{\mathcal{T}}\right)+\left\|u_{\mathcal{T}}+v_{\mathcal{T}}\right\|_{a}^{2}=\operatorname{osc}_{\mathcal{T}}^{2}\left(v_{\mathcal{T}}\right)+\left\|u_{\mathcal{T}}+v_{\mathcal{T}}\right\|_{a}^{2} .
$$

The proof is complete taking into account that $\left\|u_{\mathcal{T}}+v_{\mathcal{T}}\right\|_{a}^{2} \leq\left\|u_{\mathcal{T}}-v_{\mathcal{T}}\right\|_{a}^{2}$.

\section{Quasi-Optimality of an Adaptive Loop}

In this final section we prove the optimality of the Algorithm 1 for approximating an eigenpair corresponding to a simple eigenvalue $\lambda=\lambda_{j_{0}}$, and therefore, in this section we assume that $\lambda$ is simple. Notice that in this case, for $\mathcal{T} \in \mathbb{T}$ we have that $\tilde{E}_{\lambda}^{\mathcal{T}}=\left\{u_{\mathcal{T}},-u_{\mathcal{T}}\right\}$. Our optimality result holds provided the refinement is based on an efficient Dörfler's marking strategy (see Assumptions 5.1-5.2 below), and starting from any initial mesh. 


\subsection{Quasi-Optimality of the Total Error and Optimal Mark- ing}

In the next lemma we provide a bound for the mixed term that appears when trying to obtain an orthogonality relationship, similar to the one existing for linear problems.

Lemma 5.1. Let $\mathcal{T} \in \mathbb{T}$ be such that $h_{\mathcal{T}} \leq \mathbf{h}_{\mathbf{0}}$. If $u_{\mathcal{T}} \in \tilde{E}_{\lambda}^{\mathcal{T}}$ and $v_{\mathcal{T}} \in \mathbb{V}_{\mathcal{T}}$, there holds

$\left|a\left(\tilde{\mathcal{P}}_{\lambda} u_{\mathcal{T}}-u_{\mathcal{T}}, u_{\mathcal{T}}-v_{\mathcal{T}}\right)\right| \lesssim h_{\mathcal{T}}^{2 r}\left\|\tilde{\mathcal{P}}_{\lambda} u_{\mathcal{T}}-u_{\mathcal{T}}\right\|_{a}^{2}+\left\|\tilde{\mathcal{P}}_{\lambda} u_{\mathcal{T}}-u_{\mathcal{T}}\right\|_{a}\left\|\tilde{\mathcal{P}}_{\lambda} u_{\mathcal{T}}-v_{\mathcal{T}}\right\|_{b}$.

Proof. Let $\mathcal{T} \in \mathbb{T}$ be such that $h_{\mathcal{T}} \leq \mathbf{h}_{\mathbf{0}}$. Let $u_{\mathcal{T}} \in \tilde{E}_{\lambda}^{\mathcal{T}}$ and $\lambda_{\mathcal{T}}:=\Lambda\left(u_{\mathcal{T}}\right)$. Let us denote $u:=\tilde{\mathcal{P}}_{\lambda} u_{\mathcal{T}}$ and consider $v_{\mathcal{T}} \in \mathbb{V}_{\mathcal{T}}$ arbitrary. Since $a\left(u, u_{\mathcal{T}}-\right.$ $\left.v_{\mathcal{T}}\right)=\lambda b\left(u, u_{\mathcal{T}}-v_{\mathcal{T}}\right)$ and $a\left(u_{\mathcal{T}}, u_{\mathcal{T}}-v_{\mathcal{T}}\right)=\lambda_{\mathcal{T}} b\left(u_{\mathcal{T}}, u_{\mathcal{T}}-v_{\mathcal{T}}\right)$, it follows from Theorem 4.8 that

$$
\begin{aligned}
\left|a\left(u-u_{\mathcal{T}}, u_{\mathcal{T}}-v_{\mathcal{T}}\right)\right| & =\left|b\left(\lambda u-\lambda_{\mathcal{T}} u_{\mathcal{T}}, u_{\mathcal{T}}-v_{\mathcal{T}}\right)\right| \\
& \leq\left|b\left(\lambda u-\lambda_{\mathcal{T}} u_{\mathcal{T}}, u_{\mathcal{T}}-u\right)\right|+\left|b\left(\lambda u-\lambda_{\mathcal{T}} u_{\mathcal{T}}, u-v_{\mathcal{T}}\right)\right| \\
& =\left(\frac{\lambda+\lambda_{\mathcal{T}}}{2}\right)\left\|u-u_{\mathcal{T}}\right\|_{b}^{2}+\left|b\left(\lambda u-\lambda_{\mathcal{T}} u_{\mathcal{T}}, u-v_{\mathcal{T}}\right)\right| \\
& \lesssim h_{\mathcal{T}}^{2 r}\left\|u-u_{\mathcal{T}}\right\|_{a}^{2}+\left\|\lambda u-\lambda_{\mathcal{T}} u_{\mathcal{T}}\right\|_{b}\left\|u-v_{\mathcal{T}}\right\|_{b} .
\end{aligned}
$$

The claim of this lemma follows using that $\left\|\lambda u-\lambda_{\mathcal{T}} u_{\mathcal{T}}\right\|_{b} \lesssim\left\|u-u_{\mathcal{T}}\right\|_{a}$, which holds due to Lemma 4.2, replacing one of the discrete functions by $u$.

We now state and prove a quasi-orthogonality result that is useful for proving the Cea's Lemma below.

Theorem 5.2. There exists $\mathbf{h}_{\mathbf{4}}>0$ such that if $\mathcal{T} \in \mathbb{T}$ satisfies $h_{\mathcal{T}} \leq \mathbf{h}_{\mathbf{4}}$, then $\left\|\tilde{\mathcal{P}}_{\lambda} u_{\mathcal{T}}-u_{\mathcal{T}}\right\|_{a}^{2}+\left\|u_{\mathcal{T}}-v_{\mathcal{T}}\right\|_{a}^{2} \lesssim\left\|\tilde{\mathcal{P}}_{\lambda} u_{\mathcal{T}}-v_{\mathcal{T}}\right\|_{a}^{2}, \quad$ for all $v_{\mathcal{T}} \in \mathbb{V}_{\mathcal{T}}$ and $u_{\mathcal{T}} \in \tilde{E}_{\lambda}^{\mathcal{T}}$.

Proof. Let $\mathcal{T} \in \mathbb{T}, v_{\mathcal{T}} \in \mathbb{V}_{\mathcal{T}}, u_{\mathcal{T}} \in \tilde{E}_{\lambda}^{\mathcal{T}}$, and let us denote $u:=\tilde{\mathcal{P}}_{\lambda} u_{\mathcal{T}}$. Then,

$$
\left\|u-v_{\mathcal{T}}\right\|_{a}^{2}=\left\|u-u_{\mathcal{T}}\right\|_{a}^{2}+\left\|u_{\mathcal{T}}-v_{\mathcal{T}}\right\|_{a}^{2}+2 a\left(u-u_{\mathcal{T}}, u_{\mathcal{T}}-v_{\mathcal{T}}\right)
$$

and by Lemma 5.1,

$$
\begin{aligned}
\left|a\left(u-u_{\mathcal{T}}, u_{\mathcal{T}}-v_{\mathcal{T}}\right)\right| & \lesssim h_{\mathcal{T}}^{2 r}\left\|u-u_{\mathcal{T}}\right\|_{a}^{2}+\left\|u-u_{\mathcal{T}}\right\|_{a}\left\|u-v_{\mathcal{T}}\right\|_{b} \\
& \leq\left(\delta+h_{\mathcal{T}}^{2 r}\right)\left\|u-u_{\mathcal{T}}\right\|_{a}^{2}+\frac{1}{\delta}\left\|u-v_{\mathcal{T}}\right\|_{a}^{2}
\end{aligned}
$$

for all $\delta>0$, whenever $h_{\mathcal{T}} \leq \mathbf{h}_{\mathbf{0}}$. Thus,

$$
\left\|u-u_{\mathcal{T}}\right\|_{a}^{2}+\left\|u_{\mathcal{T}}-v_{\mathcal{T}}\right\|_{a}^{2} \lesssim\left\|u-v_{\mathcal{T}}\right\|_{a}^{2}+\left(\delta+h_{\mathcal{T}}^{2 r}\right)\left\|u-u_{\mathcal{T}}\right\|_{a}^{2}+\frac{1}{\delta}\left\|u-v_{\mathcal{T}}\right\|_{a}^{2} .
$$

Finally, we can choose $\delta>0$ and $\mathbf{h}_{\mathbf{4}}>0$ depending on the data such that

$$
\left\|u-u_{\mathcal{T}}\right\|_{a}^{2}+\left\|u_{\mathcal{T}}-v_{\mathcal{T}}\right\|_{a}^{2} \lesssim\left\|u-v_{\mathcal{T}}\right\|_{a}^{2}, \quad \text { if } h_{\mathcal{T}} \leq \mathbf{h}_{\mathbf{4}} .
$$

The following result states that the discrete eigenfunctions $u_{\mathcal{T}} \in \tilde{E}_{\lambda}^{\mathcal{T}}$ are optimal approximations (up to a constant) to the eigenspace $\tilde{E}_{\lambda}$ from within $\mathbb{V}_{\mathcal{T}}$. 
Lemma 5.3 (Cea's Lemma). Let $\mathcal{T} \in \mathbb{T}$ with $h_{\mathcal{T}} \leq \mathbf{h}_{\mathbf{4}}$ and $u_{\mathcal{T}} \in \tilde{E}_{\lambda}^{\mathcal{T}}$. Then,

$$
\operatorname{dist}_{a}^{2}\left(u_{\mathcal{T}}, \tilde{E}_{\lambda}\right)+\operatorname{osc}_{\mathcal{T}}^{2}\left(u_{\mathcal{T}}\right) \lesssim \inf _{\substack{v_{\mathcal{T}} \in \mathbb{V}_{\mathcal{T}} \\\left\|v_{\mathcal{T}}\right\|_{b}=1}}\left(\operatorname{dist}_{a}^{2}\left(v_{\mathcal{T}}, \tilde{E}_{\lambda}\right)+\operatorname{osc}_{\mathcal{T}}^{2}\left(v_{\mathcal{T}}\right)\right)
$$

Proof. Let $\mathcal{T} \in \mathbb{T}$ with $h_{\mathcal{T}} \leq \mathbf{h}_{\mathbf{4}}$, and let $u_{\mathcal{T}} \in \tilde{E}_{\lambda}^{\mathcal{T}}$. Let $v_{\mathcal{T}} \in \mathbb{V}_{\mathcal{T}}$ be such that $\left\|v_{\mathcal{T}}\right\|_{b}=1$. Without loss of generality, since $\lambda$ is a simple eigenvalue, we can assume that $u:=\tilde{\mathcal{P}}_{\lambda} v_{\mathcal{T}}=\tilde{\mathcal{P}}_{\lambda} u_{\mathcal{T}}$. Using Lemma 4.18 and Theorem 5.2 we have that

$$
\begin{aligned}
\operatorname{dist}_{a}^{2}\left(u_{\mathcal{T}}, \tilde{E}_{\lambda}\right)+\operatorname{osc}_{\mathcal{T}}^{2}\left(u_{\mathcal{T}}\right) & \lesssim\left\|u-u_{\mathcal{T}}\right\|_{a}^{2}+\operatorname{osc}_{\mathcal{T}}^{2}\left(v_{\mathcal{T}}\right)+\left\|u_{\mathcal{T}}-v_{\mathcal{T}}\right\|_{a}^{2} \\
& \lesssim\left\|u-v_{\mathcal{T}}\right\|_{a}^{2}+\operatorname{osc}_{\mathcal{T}}^{2}\left(v_{\mathcal{T}}\right) \\
& \lesssim \operatorname{dist}_{a}^{2}\left(v_{\mathcal{T}}, \tilde{E}_{\lambda}\right)+\operatorname{osc}_{\mathcal{T}}^{2}\left(v_{\mathcal{T}}\right)
\end{aligned}
$$

The following theorem states a result which is in essence a generalization of the error orthogonality that is observed in symmetric linear problems. This result becomes closer to an exact orthogonality relation as the mesh size $h_{\mathcal{T}}$ gets smaller, and will be instrumental for proving a contraction of the error.

Theorem 5.4 (Quasi-orthogonality). There exists a constant $C_{O}>0$ depending on data such that, if $\mathcal{T} \in \mathbb{T}$ satisfies $h_{\mathcal{T}} \leq \mathbf{h}_{\mathbf{1}}$ and $\mathcal{T}_{*} \in \mathbb{T}$ is a refinement of $\mathcal{T}$, then

$$
\operatorname{dist}_{a}^{2}\left(u_{\mathcal{T}_{*}}, \tilde{E}_{\lambda}\right) \leq\left(1+C_{O} h_{\mathcal{T}}^{r}\right) \operatorname{dist}_{a}^{2}\left(u_{\mathcal{T}}, \tilde{E}_{\lambda}\right)-\left\|u_{\mathcal{T}_{*}}-u_{\mathcal{T}}\right\|_{a}^{2},
$$

for $u_{\mathcal{T}} \in \tilde{E}_{\lambda}^{\mathcal{T}}$ and $u_{\mathcal{T}_{*}} \in \tilde{E}_{\lambda}^{\mathcal{T}_{*}}$ satisfying $\tilde{\mathcal{P}}_{\lambda} u_{\mathcal{T}}=\tilde{\mathcal{P}}_{\lambda} u_{\mathcal{T}_{*}}$.

Proof. Let $\mathcal{T} \in \mathbb{T}$ with $h_{\mathcal{T}} \leq \mathbf{h}_{1}$ and let $\mathcal{T}_{*} \in \mathbb{T}$ be a refinement of $\mathcal{T}$. If $u:=\tilde{\mathcal{P}}_{\lambda} u_{\mathcal{T}}=\tilde{\mathcal{P}}_{\lambda} u_{\mathcal{T}_{*}}$, we have that $\left\|u-u_{\mathcal{T}}\right\|_{a}^{2}=\left\|u-u_{\mathcal{T}_{*}}\right\|_{a}^{2}+\left\|u_{\mathcal{T}_{*}}-u_{\mathcal{T}}\right\|_{a}^{2}+$ $2 a\left(u-u_{\mathcal{T}_{*}}, u_{\mathcal{T}_{*}}-u_{\mathcal{T}}\right)$, which implies

$$
\left\|u-u_{\mathcal{T}_{*}}\right\|_{a}^{2} \leq\left\|u-u_{\mathcal{T}}\right\|_{a}^{2}-\left\|u_{\mathcal{T}_{*}}-u_{\mathcal{T}}\right\|_{a}^{2}+2\left|a\left(u-u_{\mathcal{T}_{*}}, u_{\mathcal{T}_{*}}-u_{\mathcal{T}}\right)\right| .
$$

Since $u_{\mathcal{T}} \in \mathbb{V}_{\mathcal{T}_{*}}$, Lemma 5.1 implies that

$$
\left|a\left(u-u_{\mathcal{T}_{*}}, u_{\mathcal{T}_{*}}-u_{\mathcal{T}}\right)\right| \lesssim h_{\mathcal{T}_{*}}^{2 r}\left\|u-u_{\mathcal{T}_{*}}\right\|_{a}^{2}+\left\|u-u_{\mathcal{T}_{*}}\right\|_{a}\left\|u-u_{\mathcal{T}}\right\|_{b} .
$$

Using Theorems 4.8 and 4.10 and that $h_{\mathcal{T}_{*}} \leq h_{\mathcal{T}}$ we have that

$$
\left|a\left(u-u_{\mathcal{T}_{*}}, u_{\mathcal{T}_{*}}-u_{\mathcal{T}}\right)\right| \lesssim h_{\mathcal{T}}^{r}\left\|u-u_{\mathcal{T}}\right\|_{a}^{2},
$$

and the claim of this theorem follows.

The proof of quasi-optimality is based on the use of an efficient Dörfler's marking strategy.

Assumption 5.1 (Dörfler Strategy). Let $\theta \in(0,1]$ be a marking parameter. Dörfler's marking strategy consists in selecting a minimal subset of marked elements $\mathcal{M}_{\mathcal{T}} \subset \mathcal{T}$ satisfying

$$
\eta_{\mathcal{T}}\left(u_{\mathcal{T}} ; \mathcal{M}_{\mathcal{T}}\right) \geq \theta \eta_{\mathcal{T}}\left(u_{\mathcal{T}}\right)
$$

In order to be able to prove optimality, an assumption on the marking parameter $\theta$ has to be made, related to the gap in the constants appearing in the a posteriori upper and lower bounds. 
Assumption 5.2 (The marking parameter $\theta$ ). The marking parameter $\theta$ satisfies $0<\theta<\theta_{0}$ with

$$
\theta_{0}^{2}:=\frac{C_{L}}{1+2 C_{L U}\left(1+C_{E}\right)} .
$$

The following result states the existence of a threshold $\nu$ such that, if a reduction $\nu$ is obtained in the quasi-error after some refinement of $\mathcal{T}$, then the number of refined elements is larger than the number of elements that would have been marked by Dörfler's marking strategy. We refer to this result as Optimal marking and it was first discovered for linear elliptic problems by Stevenson [22] for the exact error assuming a small oscillation, and later used for a notion of quasi-error including the oscillation terms in $[5,7]$.

Lemma 5.5 (Optimal marking). Let $\mathcal{T} \in \mathbb{T}$ with $h_{\mathcal{T}} \leq \mathbf{h}_{\mathbf{3}}$. Let $\mathcal{T}_{*} \in \mathbb{T}$ be a refinement of $\mathcal{T}$ and let $\mathcal{R}$ denote the set of the elements in $\mathcal{T}$ which are refined to obtain $\mathcal{T}_{*}$. Let Assumption 5.2 hold and let $\nu:=\frac{1}{2}\left(1-\frac{\theta^{2}}{\theta_{0}^{2}}\right)>0$. Let $u_{\mathcal{T}} \in \tilde{E}_{\lambda}^{\mathcal{T}}$ and $u_{\mathcal{T}_{*}} \in \tilde{E}_{\lambda}^{\mathcal{T}_{*}}$ such that $\operatorname{dist}_{a}\left(u_{\mathcal{T}}, \tilde{E}_{\lambda}^{\mathcal{T}_{*}}\right)=\left\|u_{\mathcal{T}}-u_{\mathcal{T}_{*}}\right\|_{a}$. If

$$
\operatorname{dist}_{a}^{2}\left(u_{\mathcal{T}_{*}}, \tilde{E}_{\lambda}\right)+\operatorname{osc}_{\mathcal{T}_{*}}^{2}\left(u_{\mathcal{T}_{*}}\right) \leq \nu\left(\operatorname{dist}_{a}^{2}\left(u_{\mathcal{T}}, \tilde{E}_{\lambda}\right)+\operatorname{osc}_{\mathcal{T}}^{2}\left(u_{\mathcal{T}}\right)\right)
$$

then

$$
\eta_{\mathcal{T}}\left(u_{\mathcal{T}} ; \mathcal{R}\right) \geq \theta \eta_{\mathcal{T}}\left(u_{\mathcal{T}}\right)
$$

Proof. Let $\mathcal{T}, \mathcal{T}_{*}, \mathcal{R}, u_{\mathcal{T}}, u_{\mathcal{T}_{*}}$ and $\nu$ be as in the assumptions of the theorem, with $h_{\mathcal{T}} \leq \mathbf{h}_{\mathbf{3}}$. Using (27) and the Global Lower Bound (Theorem 4.13) we obtain

$$
\begin{aligned}
(1-2 \nu) C_{L} \eta_{\mathcal{T}}^{2}\left(u_{\mathcal{T}}\right) & \leq(1-2 \nu)\left(\operatorname{dist}_{a}^{2}\left(u_{\mathcal{T}}, \tilde{E}_{\lambda}\right)+\operatorname{osc}_{\mathcal{T}}^{2}\left(u_{\mathcal{T}}\right)\right) \\
& \leq \operatorname{dist}_{a}^{2}\left(u_{\mathcal{T}}, \tilde{E}_{\lambda}\right)-2 \operatorname{dist}_{a}^{2}\left(u_{\mathcal{T}_{*}}, \tilde{E}_{\lambda}\right)+\operatorname{osc}_{\mathcal{T}}^{2}\left(u_{\mathcal{T}}\right)-2 \operatorname{osc}_{\mathcal{T}_{*}}^{2}\left(u_{\mathcal{T}_{*}}\right) .
\end{aligned}
$$

Since

$$
\left\|\tilde{\mathcal{P}}_{\lambda} u_{\mathcal{T}}-u_{\mathcal{T}}\right\|_{a} \leq\left\|\tilde{\mathcal{P}}_{\lambda} u_{\mathcal{T}_{*}}-u_{\mathcal{T}_{*}}\right\|_{a}+\left\|u_{\mathcal{T}_{*}}-u_{\mathcal{T}}\right\|_{a}
$$

we have that $\operatorname{dist}_{a}\left(u_{\mathcal{T}}, \tilde{E}_{\lambda}\right) \leq \operatorname{dist}_{a}\left(u_{\mathcal{T}_{*}}, \tilde{E}_{\lambda}\right)+\left\|u_{\mathcal{T}_{*}}-u_{\mathcal{T}}\right\|_{a}$, and therefore,

$$
\operatorname{dist}_{a}^{2}\left(u_{\mathcal{T}}, \tilde{E}_{\lambda}\right) \leq 2 \operatorname{dist}_{a}^{2}\left(u_{\mathcal{T}_{*}}, \tilde{E}_{\lambda}\right)+2\left\|u_{\mathcal{T}_{*}}-u_{\mathcal{T}}\right\|_{a}^{2} .
$$

On the other hand, Proposition 4.17 implies

$$
\operatorname{osc}_{\mathcal{T}}^{2}\left(u_{\mathcal{T}} ; \mathcal{T} \cap \mathcal{T}_{*}\right)-2 \operatorname{osc}_{\mathcal{T}_{*}}^{2}\left(u_{\mathcal{T}_{*}} ; \mathcal{T} \cap \mathcal{T}_{*}\right) \leq 2 C_{E}\left\|u_{\mathcal{T}_{*}}-u_{\mathcal{T}}\right\|_{a}^{2},
$$

and there holds $\operatorname{osc}_{\mathcal{T}}^{2}\left(u_{\mathcal{T}} ; T\right) \leq \eta_{\mathcal{T}}^{2}\left(u_{\mathcal{T}} ; T\right)$, for $T \in \mathcal{R}$. Recalling that $\mathcal{R}=\mathcal{T} \backslash \mathcal{T}_{*}$, for the oscillation terms we obtain

$$
\operatorname{osc}_{\mathcal{T}}^{2}\left(u_{\mathcal{T}}\right)-2 \operatorname{osc}_{\mathcal{T}_{*}}^{2}\left(u_{\mathcal{T}_{*}}\right) \leq 2 C_{E}\left\|u_{\mathcal{T}_{*}}-u_{\mathcal{T}}\right\|_{a}^{2}+\eta_{\mathcal{T}}^{2}\left(u_{\mathcal{T}} ; \mathcal{R}\right) .
$$

Now, this last inequality and (28) yield

$$
(1-2 \nu) C_{L} \eta_{\mathcal{T}}^{2}\left(u_{\mathcal{T}}\right) \leq 2\left\|u_{\mathcal{T}}-u_{\mathcal{T}_{*}}\right\|_{a}^{2}+2 C_{E}\left\|u_{\mathcal{T}}-u_{\mathcal{T}_{*}}\right\|_{a}^{2}+\eta_{\mathcal{T}}^{2}\left(u_{\mathcal{T}} ; \mathcal{R}\right),
$$

whence the Localized Upper Bound (Theorem 4.15) implies that

$$
\begin{aligned}
(1-2 \nu) C_{L} \eta_{\mathcal{T}}^{2}\left(u_{\mathcal{T}}\right) & \leq 2\left(1+C_{E}\right) C_{L U} \eta_{\mathcal{T}}^{2}\left(u_{\mathcal{T}} ; \mathcal{R}\right)+\eta_{\mathcal{T}}^{2}\left(u_{\mathcal{T}} ; \mathcal{R}\right) \\
& =\left(1+2 C_{L U}\left(1+C_{E}\right)\right) \eta_{\mathcal{T}}^{2}\left(u_{\mathcal{T}} ; \mathcal{R}\right)
\end{aligned}
$$


Therefore,

$$
\frac{(1-2 \nu) C_{L}}{1+2 C_{L U}\left(1+C_{E}\right)} \eta_{\mathcal{T}}^{2}\left(u_{\mathcal{T}}\right) \leq \eta_{\mathcal{T}}^{2}\left(u_{\mathcal{T}} ; \mathcal{R}\right)
$$

which implies the claim since the definition of $\nu$ implies $\theta^{2}=\frac{(1-2 \nu) C_{L}}{1+2 C_{L U}\left(1+C_{E}\right)}$.

Following the ideas in $[5,7]$ we now prove that Dörlfler's strategy yields a contraction for the sum of energy error plus a scaled error estimator; starting at some iterate $\mathbf{k}_{\mathbf{0}}$.

Theorem 5.6 (A contraction property). Let us suppose that the continuous problem (2) satisfies the Non-Degeneracy Assumption 3.1, let $\left\{\left(\lambda_{k}, u_{k}\right)\right\}_{k \in \mathbb{N}_{0}}$ denote the sequence of computed eigenpairs obtained through Algorithm 1, where we consider the Dörfler's Strategy (Assumption 5.1) for the module MARK. Then, there exist constants $\gamma>0$, and $0<\alpha<1$, depending on the data, and $\mathbf{k}_{\mathbf{0}} \in \mathbb{N}$, such that

$$
\operatorname{dist}_{a}^{2}\left(u_{k+1}, \tilde{E}_{\lambda}\right)+\gamma \eta_{k+1}^{2} \leq \alpha^{2}\left(\operatorname{dist}_{a}^{2}\left(u_{k}, \tilde{E}_{\lambda}\right)+\gamma \eta_{k}^{2}\right), \quad \text { for } k \geq \mathbf{k}_{\mathbf{0}},
$$

where $\eta_{k}:=\eta_{T_{k}}\left(u_{k}\right)$ denotes the global error estimator.

Proof. We define the auxiliary sequence $\left\{w_{k}\right\}_{k \in \mathbb{N}_{0}}$ given by $w_{0}:=u_{0}$, and $w_{k}=u_{k}$ or $-u_{k}$ in order to satisfy $\tilde{\mathcal{P}}_{\lambda} w_{k}=\tilde{\mathcal{P}}_{\lambda} u_{0}$, for all $k \in \mathbb{N}$. Notice that $\operatorname{dist}_{a}\left(w_{k}, \tilde{E}_{\lambda}\right)=\operatorname{dist}_{a}\left(u_{k}, \tilde{E}_{\lambda}\right), \operatorname{osc}_{\mathcal{T}_{k}}\left(w_{k}\right)=\operatorname{osc}_{\mathcal{T}_{k}}\left(u_{k}\right)$, and also $w_{k}$ and $u_{k}$ both yield the same a posteriori error estimators. Therefore, we can interpret the algorithm to have produced the sequence $\left\{w_{k}\right\}_{k \in \mathbb{N}_{0}}$ instead of $\left\{u_{k}\right\}_{k \in \mathbb{N}_{0}}$, since the sequence of meshes, estimators and errors would coincide.

Let $u:=\tilde{\mathcal{P}}_{\lambda} u_{0}$. We will use the notation $e_{k}:=\operatorname{dist}_{a}\left(u_{k}, \tilde{E}_{\lambda}\right)=\operatorname{dist}_{a}\left(w_{k}, \tilde{E}_{\lambda}\right)=$ $\left\|u-w_{k}\right\|_{a}$, and $\eta_{k}\left(\mathcal{M}_{k}\right):=\eta_{\mathcal{T}_{k}}\left(u_{k} ; \mathcal{M}_{k}\right)$. We combine the quasi-orthogonality relationship (26) with Proposition 4.16 with $\mathcal{T}=\mathcal{T}_{k}$ and $\mathcal{T}_{*}=\mathcal{T}_{k+1}$ to write

$$
\begin{aligned}
e_{k+1}^{2}+\gamma \eta_{k+1}^{2} \leq & e_{k}^{2}-\left\|w_{k+1}-w_{k}\right\|_{a}^{2}+C_{O} h_{\mathcal{T}_{k}}^{r} e_{k}^{2} \\
& +(1+\delta) \gamma\left\{\eta_{k}^{2}-c \eta_{k}^{2}\left(\mathcal{M}_{k}\right)\right\}+\left(1+\delta^{-1}\right) C_{E} \gamma\left\|w_{k+1}-w_{k}\right\|_{a}^{2},
\end{aligned}
$$

whenever $h_{\mathcal{T}_{k}} \leq \mathbf{h}_{\mathbf{1}}$. Setting $\gamma:=\frac{1}{\left(1+\delta^{-1}\right) C_{E}}$, we have

$$
e_{k+1}^{2}+\gamma \eta_{k+1}^{2} \leq e_{k}^{2}+(1+\delta) \gamma\left\{\eta_{k}^{2}-c \eta_{k}^{2}\left(\mathcal{M}_{k}\right)\right\}+C_{O} h_{\mathcal{T}_{k}}^{r} e_{k}^{2} .
$$

Dörfler's strategy implies that $\eta_{k}\left(\mathcal{M}_{k}\right) \geq \theta \eta_{k}$ and so

$$
\begin{aligned}
e_{k+1}^{2}+\gamma \eta_{k+1}^{2} & \leq e_{k}^{2}+(1+\delta) \gamma \eta_{k}^{2}-(1+\delta) \gamma c \theta^{2} \eta_{k}^{2}+C_{O} h_{\mathcal{T}_{k}}^{r} e_{k}^{2} \\
& \leq e_{k}^{2}+(1+\delta) \gamma \eta_{k}^{2}-(1+\delta) \gamma \frac{c \theta^{2}}{2} \eta_{k}^{2}-(1+\delta) \gamma \frac{c \theta^{2}}{2} \eta_{k}^{2}+C_{O} h_{\mathcal{T}_{k}}^{r} e_{k}^{2} .
\end{aligned}
$$

Now, using the Global Upper Bound (Theorem 4.14) and that $\gamma(1+\delta)=\frac{\delta}{C_{E}}$ we have

$$
e_{k+1}^{2}+\gamma \eta_{k+1}^{2} \leq e_{k}^{2}-\frac{\delta c \theta^{2}}{2 C_{U} C_{E}} e_{k}^{2}+(1+\delta) \gamma\left(1-\frac{c \theta^{2}}{2}\right) \eta_{k}^{2}+C_{O} h_{\mathcal{T}_{k}}^{r} e_{k}^{2},
$$

if $h_{\mathcal{T}_{k}} \leq \min \left(\mathbf{h}_{\mathbf{1}}, \mathbf{h}_{\mathbf{2}}\right)$. If we define

$$
\alpha_{1}^{2}(\delta):=\left(1-\frac{\delta c \theta^{2}}{2 C_{U} C_{E}}\right), \quad \alpha_{2}^{2}(\delta):=\left(1-\frac{c \theta^{2}}{2}\right)(1+\delta)
$$


then

$$
e_{k+1}^{2}+\gamma \eta_{k+1}^{2} \leq \alpha_{1}^{2}(\delta) e_{k}^{2}+\gamma \alpha_{2}^{2}(\delta) \eta_{k}^{2}+C_{O} h_{\mathcal{T}_{k}}^{r} e_{k}^{2} .
$$

Now, the parameter $\delta$ can be chosen such that

$$
0<\alpha:=\max \left\{\alpha_{1}(\delta), \alpha_{2}(\delta)\right\}<1,
$$

and we obtain

$$
e_{k+1}^{2}+\gamma \eta_{k+1}^{2} \leq \alpha^{2}\left(e_{k}^{2}+\gamma \eta_{k}^{2}\right)+C_{O} h_{\mathcal{T}_{k}}^{r} e_{k}^{2} .
$$

By Lemma 3.12, we have that $h_{\mathcal{T}_{k}} \rightarrow 0$ and thus, we can choose $\mathbf{k}_{\mathbf{0}} \in \mathbb{N}$ large enough such that $h_{\mathcal{T}_{\mathbf{k}_{\mathbf{0}}}} \leq \min \left(\mathbf{h}_{\mathbf{1}}, \mathbf{h}_{\mathbf{2}}\right)$ and $C_{O} h_{\mathcal{T}_{k}}^{r} \leq \frac{\left(1-\alpha^{2}\right)}{2}$, for all $k \geq \mathbf{k}_{\mathbf{0}}$. Redefining $\alpha^{2}:=\frac{1+\alpha^{2}}{2}$, we obtain

$$
e_{k+1}^{2}+\gamma \eta_{k+1}^{2} \leq \alpha^{2}\left(e_{k}^{2}+\gamma \eta_{k}^{2}\right), \quad \text { for all } k \geq \mathbf{k}_{\mathbf{0}}
$$

\subsection{Quasi-optimal cardinality of AFEM and Main Result}

In this section we first define what we understand by quasi-optimality of AFEM for the Steklov eigenvalue problem considered in this article, and prove the second main result of this paper. For $N \in \mathbb{N}$, let $\mathbb{T}_{N}$ be the set of all possible conforming triangulations generated by refinement from $\mathcal{T}_{0}$ with at most $N$ elements more than $\mathcal{T}_{0}$, i.e.,

$$
\mathbb{T}_{N}:=\left\{\mathcal{T} \in \mathbb{T}: \quad \# \mathcal{T}-\# \mathcal{T}_{0} \leq N\right\} .
$$

For an eigenvalue $\lambda$ of the continuous problem (2), the quality of the best approximation to the total error in the set $\mathbb{T}_{N}$ is given by

$$
\sigma(\lambda, \mathbf{D} ; N):=\inf _{\mathcal{T} \in \mathbb{T}_{N}} \inf _{\substack{v_{\mathcal{T}} \in \mathbb{V}_{\mathcal{T}} \\\left\|v_{\mathcal{T}}\right\|_{b}=1}}\left(\operatorname{dist}_{a}^{2}\left(v_{\mathcal{T}}, \tilde{E}_{\lambda}\right)+\operatorname{osc}_{\mathcal{T}}^{2}\left(v_{\mathcal{T}}\right)\right)^{\frac{1}{2}},
$$

where $\mathbf{D}:=\{\mathcal{A}, c, \rho, \Omega\}$ is the set of problem data. For $s>0$, we say that $(\lambda, \mathbf{D}) \in \mathbb{A}_{s}$ if

$$
|(\lambda, \mathbf{D})|_{s}:=\sup _{N \in \mathbb{N}}\left(N^{s} \sigma(\lambda, \mathbf{D} ; N)\right)<\infty .
$$

In other words, the eigenvalue belongs to the class $\mathbb{A}_{s}$ if its eigenfunctions can be ideally approximated with adaptive meshes at a rate $(D O F s)^{-s}$. The study of classes of functions that will yield such rates is beyond the scope of this article. Some results along this direction can be found in [4, 11, 12].

The following result, proved in $[22,5]$, provides a bound for the complexity of the overlay of two triangulations $\mathcal{T}^{1}$ and $\mathcal{T}^{2}$ obtained as refinements of $\mathcal{T}_{0}$.

Lemma 5.7 (Overlay of triangulations). For $\mathcal{T}^{1}, \mathcal{T}^{2} \in \mathbb{T}$ the overlay $\mathcal{T}:=\mathcal{T}^{1} \oplus$ $\mathcal{T}^{2} \in \mathbb{T}$, defined as the smallest admissible triangulation which is a refinement of $\mathcal{T}^{1}$ and $\mathcal{T}^{2}$, satisfies

$$
\# \mathcal{T} \leq \# \mathcal{T}^{1}+\# \mathcal{T}^{2}-\# \mathcal{T}_{0}
$$

The next lemma is essential for proving the main result below (see Theorem 5.10). 
Lemma 5.8 (Cardinality of $\mathcal{M}_{k}$ ). Let us suppose that the continuous problem (2) satisfies the Non-Degeneracy Assumption 3.1, let $\left\{\left(\lambda_{k}, u_{k}\right)\right\}_{k \in \mathbb{N}_{0}}$ denote the sequence of computed eigenpairs obtained through Algorithm 1, where we consider the Dörfler's Strategy (Assumption 5.1) for the module MARK with the marking parameter $\theta$ satisfying Assumption 5.2. Then, if $(\lambda, \boldsymbol{D}) \in \mathbb{A}_{s}$, there exists $\mathbf{k}_{\mathbf{1}} \in \mathbb{N}$ such that

$$
\# \mathcal{M}_{k} \lesssim|(\lambda, \boldsymbol{D})|_{s}^{\frac{1}{s}}\left(\operatorname{dist}_{a}^{2}\left(u_{k}, \tilde{E}_{\lambda}\right)+\operatorname{osc}_{\mathcal{T}_{k}}^{2}\left(u_{k}\right)\right)^{-\frac{1}{2 s}}, \quad \text { for all } k \geq \mathbf{k}_{\mathbf{1}} .
$$

Proof. Let $k \in \mathbb{N}_{0}$ be fixed. Let $\epsilon=\epsilon(k)>0$ be a tolerance to be determined later. As $(\lambda, \mathbf{D}) \in \mathbb{A}_{s}$, there exist a triangulation $\mathcal{T}_{\epsilon} \in \mathbb{T}$ and a function $v_{\epsilon} \in \mathbb{V}_{\mathcal{T}_{\epsilon}}$ with $\left\|v_{\epsilon}\right\|_{b}=1$ such that

$$
\# \mathcal{T}_{\epsilon}-\# \mathcal{T}_{0} \leq|(\lambda, \mathbf{D})|_{s}^{\frac{1}{s}} \epsilon^{-\frac{1}{s}}, \quad \text { and } \quad \operatorname{dist}_{a}^{2}\left(v_{\epsilon}, \tilde{E}_{\lambda}\right)+\operatorname{osc}_{\mathcal{T}_{\epsilon}}^{2}\left(v_{\epsilon}\right) \leq \epsilon^{2} .
$$

Let $\mathcal{T}_{*}:=\mathcal{T}_{\epsilon} \oplus \mathcal{T}_{k}$ be the overlay of $\mathcal{T}_{\epsilon}$ and $\mathcal{T}_{k}$, and $u_{\mathcal{T}_{*}} \in \tilde{E}_{\lambda}^{\mathcal{T}_{*}}$ such that $\operatorname{dist}_{a}\left(u_{k}, \tilde{E}_{\lambda}^{\mathcal{T}_{*}}\right)=\left\|u_{k}-u_{\mathcal{T}_{*}}\right\|_{a}$. Since $v_{\epsilon} \in \mathbb{V}_{\mathcal{T}_{*}}$, we have that $\operatorname{osc}_{\mathcal{T}_{\epsilon}}\left(v_{\epsilon}\right) \geq$ $\operatorname{osc}_{\mathcal{T}_{*}}\left(v_{\epsilon}\right)$. By Cea's Lemma (Lemma 5.3) we have that

$$
\operatorname{dist}_{a}^{2}\left(u_{\mathcal{T}_{*}}, \tilde{E}_{\lambda}\right)+\operatorname{osc}_{\mathcal{T}_{*}}^{2}\left(u_{\mathcal{T}_{*}}\right) \lesssim \operatorname{dist}_{a}^{2}\left(v_{\epsilon}, \tilde{E}_{\lambda}\right)+\operatorname{osc}_{\mathcal{T}_{\epsilon}}^{2}\left(v_{\epsilon}\right) \leq \epsilon^{2},
$$

if $h_{\mathcal{T}_{*}} \leq \mathbf{h}_{\mathbf{4}}$. We can now select $\epsilon$ such that

$$
\operatorname{dist}_{a}^{2}\left(u_{\mathcal{T}_{*}}, \tilde{E}_{\lambda}\right)+\operatorname{osc}_{\mathcal{T}_{*}}^{2}\left(u_{\mathcal{T}_{*}}\right) \leq \nu\left(\operatorname{dist}_{a}^{2}\left(u_{k}, \tilde{E}_{\lambda}\right)+\operatorname{osc}_{\mathcal{T}_{k}}^{2}\left(u_{k}\right)\right),
$$

where $\nu$ is the constant given by Lemma 5.5. Thus, this lemma yields

$$
\eta_{\mathcal{T}_{k}}^{2}\left(u_{k} ; \mathcal{R}\right) \geq \theta^{2} \eta_{\mathcal{T}_{k}}^{2}\left(u_{k}\right)
$$

whenever $h_{\mathcal{T}_{k}} \leq \mathbf{h}_{\mathbf{3}}$, if $\mathcal{R}$ is the set of elements in $\mathcal{T}_{k}$ which are refined to obtain $\mathcal{T}_{*}$. Recalling that $\mathcal{M}_{k}$ is the minimal subset of $\mathcal{T}_{k}$ satisfying Dörfler's property, using Lemma 5.7 and taking into account the choice of $\epsilon$ we obtain that

$$
\begin{aligned}
\# \mathcal{M}_{k} & \leq \# \mathcal{R} \leq \# \mathcal{T}_{*}-\# \mathcal{T}_{k} \leq \# \mathcal{T}_{\epsilon}-\# \mathcal{T}_{0} \\
& \leq|(\lambda, \mathbf{D})|_{s}^{\frac{1}{s}} \epsilon^{-\frac{1}{s}} \lesssim|(\lambda, \mathbf{D})|_{s}^{\frac{1}{s}}\left(\operatorname{dist}_{a}^{2}\left(u_{k}, \tilde{E}_{\lambda}\right)+\operatorname{osc}_{\mathcal{T}_{k}}^{2}\left(u_{k}\right)\right)^{-\frac{1}{2 s}},
\end{aligned}
$$

whenever $h_{\mathcal{T}_{k}} \leq \min \left(\mathbf{h}_{\mathbf{3}}, \mathbf{h}_{\mathbf{4}}\right)$. Since $h_{\mathcal{T}_{k}} \rightarrow 0$, we can choose $\mathbf{k}_{\mathbf{1}} \geq \mathbf{k}_{\mathbf{0}}$ large enough such that $h_{\mathcal{T}_{k}} \leq \min \left(\mathbf{h}_{\mathbf{3}}, \mathbf{h}_{\mathbf{4}}\right)$, when $k \geq \mathbf{k}_{\mathbf{1}}$, and the assertion of this lemma follows.

The next result bounds the complexity of a mesh $\mathcal{T}_{k}$ in terms of the number of elements that were marked from the beginning of the iterative process, assuming that all the meshes were obtained by the bisection algorithm of [23], and that the initial mesh was properly labeled (condition (b) of Section 4 in [23]).

Lemma 5.9 (Complexity of REFINE). Let us assume that $\mathcal{T}_{0}$ satisfies the labeling condition (b) of Section 4 in Ref. [23], and consider the sequence $\left\{\mathcal{T}_{k}\right\}_{k \in \mathbb{N}_{0}}$ of refinements of $\mathcal{T}_{0}$ where $\mathcal{T}_{k+1}:=\operatorname{REFINE}\left(\mathcal{T}_{k}, \mathcal{M}_{k}\right)$ with $\mathcal{M}_{k} \subset \mathcal{T}_{k}$. Then, there exists a constant solely depending on $\mathcal{T}_{0}$ and the number of refinements $n$ performed by REFINE to marked elements, such that

$$
\# \mathcal{T}_{k}-\# \mathcal{T}_{0} \lesssim \sum_{i=0}^{k-1} \# \mathcal{M}_{i}, \quad \text { for all } k \in \mathbb{N} .
$$


The next result will use Lemma 5.9 and is a consequence of the Global Lower Bound (Theorem 4.13), the bound for the cardinality of $\mathcal{M}_{k}$ given by Lemma 5.8 and the contraction property of Theorem 5.6. This is the second main result of the paper.

Theorem 5.10 (Quasi-optimal complexity). Let us suppose that the continuous problem (2) satisfies the Non-Degeneracy Assumption 3.1, let $\left\{\left(\lambda_{k}, u_{k}\right)\right\}_{k \in \mathbb{N}_{0}}$ denote the sequence of computed eigenpairs obtained through Algorithm 1, where we consider the Dörfler's Strategy (Assumption 5.1) for the module MARK with the marking parameter $\theta$ satisfying Assumption 5.2, and let $\mathbf{k}_{\mathbf{1}}$ be as in Lemma 5.8. Let $\mathcal{T}_{0}$ be labeled to satisfy the assumption of Lemma 5.9. If $(\lambda, \boldsymbol{D}) \in \mathbb{A}_{s}$, then

$$
\left(\operatorname{dist}_{a}^{2}\left(u_{k}, \tilde{E}_{\lambda}\right)+\operatorname{osc}_{\mathcal{T}_{k}}^{2}\left(u_{k}\right)\right)^{\frac{1}{2}} \lesssim|(\lambda, \boldsymbol{D})|_{s}\left(\# \mathcal{T}_{k}-\# \mathcal{T}_{\mathbf{k}_{\mathbf{1}}}\right)^{-s}, \quad \text { for } k>\mathbf{k}_{\mathbf{1}} \text {. }
$$

Proof. Let $k>\mathbf{k}_{\mathbf{1}}$. By Lemmas 5.8 and 5.9 we have

$$
\begin{aligned}
\# \mathcal{T}_{k}-\# \mathcal{T}_{\mathbf{k}_{\mathbf{1}}} & \lesssim \sum_{i=\mathbf{k}_{\mathbf{1}}}^{k-1} \# \mathcal{M}_{i} \lesssim|(\lambda, \mathbf{D})|_{s}^{\frac{1}{s}} \sum_{i=\mathbf{k}_{\mathbf{1}}}^{k-1}\left(\operatorname{dist}_{a}^{2}\left(u_{i}, \tilde{E}_{\lambda}\right)+\operatorname{osc}_{\mathcal{T}_{i}}^{2}\left(u_{i}\right)\right)^{-\frac{1}{2 s}} \\
& \lesssim|(\lambda, \mathbf{D})|_{s}^{\frac{1}{s}} \sum_{i=\mathbf{k}_{\mathbf{1}}}^{k-1}\left(\operatorname{dist}_{a}^{2}\left(u_{i}, \tilde{E}_{\lambda}\right)+\gamma \eta_{\mathcal{T}_{i}}^{2}\left(u_{i}\right)\right)^{-\frac{1}{2 s}}
\end{aligned}
$$

where for the last inequality we have used the Global Lower Bound (Theorem 4.13). If we define $z_{i}^{2}:=\operatorname{dist}_{a}^{2}\left(u_{i}, \tilde{E}_{\lambda}\right)+\gamma \eta_{\mathcal{T}_{i}}^{2}\left(u_{i}\right)$, the contraction property (Theorem 5.6) implies that $z_{i+1} \leq \alpha z_{i}$ and therefore, $z_{i}^{-\frac{1}{s}} \leq \alpha^{\frac{1}{s}} z_{i+1}^{-\frac{1}{s}}$. Since $\alpha<1$, we obtain that

$$
\sum_{i=\mathbf{k}_{1}}^{k-1} z_{i}^{-\frac{1}{s}} \leq \sum_{i=0}^{\infty}\left(\alpha^{\frac{1}{s}}\right)^{i} z_{k}^{-\frac{1}{s}} \lesssim z_{k}^{-\frac{1}{s}}
$$

and from (30) it follows that

$$
\# \mathcal{T}_{k}-\# \mathcal{T}_{\mathbf{k}_{1}} \lesssim|(\lambda, \mathbf{D})|_{s}^{\frac{1}{s}}\left(\operatorname{dist}_{a}^{2}\left(u_{k}, \tilde{E}_{\lambda}\right)+\gamma \eta_{\mathcal{T}_{k}}^{2}\left(u_{k}\right)\right)^{-\frac{1}{2 s}}
$$

Recalling that $\operatorname{osc}_{\mathcal{T}_{k}}\left(u_{k}\right) \lesssim \eta_{\mathcal{T}_{k}}\left(u_{k}\right)$, this inequality can be written as

$$
\# \mathcal{T}_{k}-\# \mathcal{T}_{\mathbf{k}_{\mathbf{1}}} \lesssim|(\lambda, \mathbf{D})|_{s}^{\frac{1}{s}}\left(\operatorname{dist}_{a}^{2}\left(u_{k}, \tilde{E}_{\lambda}\right)+\operatorname{osc}_{\mathcal{T}_{k}}^{2}\left(u_{k}\right)\right)^{-\frac{1}{2 s}}
$$

and we finally obtain the desired estimate raising to the $s$-power and reordering.

The right-hand side of (29) is not quite what we desire yet, since it appears $\# \mathcal{T}_{\mathbf{k}_{\mathbf{1}}}$ where it should appear $\# \mathcal{T}_{0}$, but this can be fixed. In fact, let $\mathbf{k}_{\mathbf{2}} \in \mathbb{N}$ be large enough such that $\# \mathcal{T}_{k} \geq 2 \# \mathcal{T}_{\mathbf{k}_{\mathbf{1}}}-\# \mathcal{T}_{0}$, for all $k \geq \mathbf{k}_{\mathbf{2}}$. Then

$\# \mathcal{T}_{k}-\# \mathcal{T}_{0}=\left(\# \mathcal{T}_{k}-\# \mathcal{T}_{\mathbf{k}_{\mathbf{1}}}\right)+\left(\# \mathcal{T}_{\mathbf{k}_{\mathbf{1}}}-\# \mathcal{T}_{0}\right) \leq 2\left(\# \mathcal{T}_{k}-\# \mathcal{T}_{\mathbf{k}_{1}}\right), \quad$ for all $k \geq \mathbf{k}_{\mathbf{2}}$, and therefore,

$$
\left(\# \mathcal{T}_{k}-\# \mathcal{T}_{\mathbf{k}_{\mathbf{1}}}\right)^{-s} \leq 2^{s}\left(\# \mathcal{T}_{k}-\# \mathcal{T}_{0}\right)^{-s}, \quad \text { for all } k \geq \mathbf{k}_{\mathbf{2}}
$$

Finally, after possibly changing the constant the main result becomes

Corollary 5.11. Under the assumptions of Theorem 5.10, there holds

$$
\left(\operatorname{dist}_{a}^{2}\left(u_{k}, \tilde{E}_{\lambda}\right)+\operatorname{osc}_{\mathcal{T}_{k}}^{2}\left(u_{k}\right)\right)^{\frac{1}{2}} \lesssim|(\lambda, \boldsymbol{D})|_{s}\left(\# \mathcal{T}_{k}-\# \mathcal{T}_{0}\right)^{-s}, \quad \text { for all } k \in \mathbb{N} .
$$




\section{References}

[1] María G. Armentano and Claudio Padra, A posteriori error estimates for the Steklov eigenvalue problem, Appl. Numer. Math. 58 (2008), 593-601.

[2] Ivo Babuška and John E. Osborn, Eigenvalue problems, Handbook of numerical analysis II (1991), 641-787.

[3] Alfredo Bermúdez, Rodolfo Rodríguez and Duarte Santamarina, A finite element solution of an added mass formulation for coupled fluid-solid vibrations, Numerische Mathematik 87 (2000), 201-227.

[4] Peter Binev, Wolfgang Dahmen, Ronald DeVore, Pencho Petrushev, Approximation Classes for Adaptive Methods, Serdica Math. J. 28 (2002), 391-416.

[5] J. Manuel Cascón, Christian Kreuzer, Ricardo H. Nochetto, and Kunibert G. Siebert, Quasi-optimal convergence rate for an adaptive finite element method, SIAM J. Numer. Anal. 46 (2008), 2524-2550.

[6] Carlos Conca, Jacques Planchard, and Muthusamy Vanninathan, Fluids and periodic structures, RAM: Research in Applied Mathematics 38 (1995), John Wiley \& Sons Ltd., Chichester.

[7] Xiaoying Dai, Jinchao Xu, and Aihui Zhou, Convergence and optimal complexity of adaptive finite element eigenvalue computations, Numer. Math. 110 (2008), 313-355.

[8] Willy Dörfler, A convergent adaptive algorithm for Poisson's equation, SIAM J. Numer. Anal. 33 (1996), 1106-1124.

[9] Ricardo G. Durán, Claudio Padra, and Rodolfo Rodríguez, A posteriori error estimates for the finite element approximation of eigenvalue problems, Math. Models Methods Appl. Sci. 13 (2003), 1219-1229.

[10] Eduardo M. Garau, Pedro Morin, and Carlos Zuppa, Convergence of adaptive finite element methods for eigenvalue problems, M3AS: Mathematical Models and Methods in Applied Sciences 19 (2009), 721-747.

[11] Fernando D. Gaspoz, Pedro Morin, Convergence rates for adaptive finite elements, IMA Journal of Numerical Analysis (2008); doi: 10.1093/imanum/drn039.

[12] Fernando D. Gaspoz, Pedro Morin, Approximation classes for adaptive higher order finite element approximation, in preparation (2009).

[13] Stefano Giani, Convergence adaptive finite element methods for elliptic eigenvalue problems with application to photonic crystal fibers (pcfs), $\mathrm{PhD}$ Thesis, University of Bath (2008).

[14] Stefano Giani and Ivan G. Graham, A convergent adaptive method for elliptic eigenvalue problems, SIAM J. Numer. Anal. 47 (2009), 1067-1091.

[15] Pierre Grisvard, Elliptic Problems in Nonsmooth Domains, Pitman, Boston (1985). 
[16] Qing Han, Singular sets of solutions to elliptic equations, Indiana Univ. Math. J. 43 (1994), 983-1002.

[17] Pedro Morin, Kunibert G. Siebert, and Andreas Veeser, A basic convergence result for conforming adaptive finite elements, Math. Models Methods Appl. Sci. 18 (2008), 707-737.

[18] Giuseppe Savaré, Regularity results for elliptic equations in Lipschitz domains, J. Funct. Anal. 152 (1998), 176-201.

[19] Alfred Schmidt and Kunibert G. Siebert, Design of adaptive finite element software, Lecture Notes in Computational Science and Engineering 42 (2005), Springer-Verlag, Berlin, The finite element toolbox ALBERTA, With 1 CD-ROM (Unix/Linux).

[20] L. Ridgway Scott and Shangyou Zhang, Finite element interpolation of nonsmooth functions satisfying boundary conditions, Math. Comp. 54 (1990), 483-493.

[21] Kunibert G. Siebert, A convergence proof for adaptive finite elements without lower bound, Preprint Universität Duisburg-Essen (2008).

[22] Rob Stevenson, Optimality of a standard adaptive finite element method, Found. Comput. Math. 7 (2007), 245-269.

[23] Rob Stevenson, The completion of locally refined simplicial partitions created by bisection, Math. Comp. 77 (2008), 227-241 (electronic).

[24] Gilbert Strang and George J. Fix, An analysis of the finite element method, Prentice-Hall Inc., Englewood Cliffs, N. J. (1973), Prentice-Hall Series in Automatic Computation.

[25] Carlos Zuppa, A posteriori error estimates for the finite element approximation of steklov eigenvalue problem, Mecánica Computacional 26 (2007), $724-735$.

\section{Affiliations}

Eduardo M. Garau: Consejo Nacional de Investigaciones Científicas y Técnicas and Universidad Nacional del Litoral, Argentina, egarau@santafe-conicet.gov.ar. Partially supported by CONICET (Argentina) through Grant PIP 112-20080102182, and Universidad Nacional del Litoral through Grant CAI+D 008-054 and CAI+D PI 062-312.

Address: IMAL, Güemes 3450. S3000GLN Santa Fe, ARGENTINA

Pedro Morin: Consejo Nacional de Investigaciones Científicas y Técnicas and Universidad Nacional del Litoral, Argentina, pmorin@santafe-conicet.gov . ar. Partially supported by CONICET (Argentina) through Grant PIP 112-20080102182, and Universidad Nacional del Litoral through Grant CAI+D 008-054 and CAI+D PI 062-312.

Address: IMAL, Güemes 3450. S3000GLN Santa Fe, ARGENTINA 